\title{
Genetic variation determines which feedbacks drive and alter predator-prey eco-evolutionary cycles
}

\author{
Michael H. Cortez ${ }^{1}$ \\ Department of Mathematics and Statistics, Utah State University, Logan, Utah 84322 USA
}

\begin{abstract}
Evolution can alter the ecological dynamics of communities, but the effects depend on the magnitudes of standing genetic variation in the evolving species. Using an eco-coevolutionary predator-prey model, I identify how the magnitudes of prey and predator standing genetic variation determine when ecological, evolutionary, and eco-evolutionary feedbacks influence system stability and the phase lags in predator-prey cycles. Here, feedbacks are defined by subsystems, i.e., the dynamics of a subset of the components of the whole system when the other components are held fixed; ecological (evolutionary) feedbacks involve the direct and indirect effects between population densities (species traits) and eco-evolutionary feedbacks involve the direct and indirect effects between population densities and traits. When genetic variation is low in both species, ecological feedbacks and eco-evolutionary feedbacks involving either the predator or the prey trait have the strongest effects on system stability, when genetic variation is high in one species, evolutionary and eco-evolutionary feedbacks involving that species' trait have the strongest effects, and, when genetic variation is high in both species, evolutionary feedbacks involving one or both traits and eco-coevolutionary feedbacks involving both traits have the strongest effects. I present the biological conditions under which each feedback can destabilize the whole system and cause predator-prey cycles. Predator-prey cycles can also arise when all feedbacks are stabilizing. This counterintuitive outcome occurs when feedbacks involving many variables are more stabilizing than feedbacks involving fewer variables or vice versa. I also identify how the indirect effects of prey and predator density on the predator dynamics (mediated by evolutionary responses in one or both species) alter the phase lags in predator-prey cycles. I present conditions under which the trait-mediated indirect effects introduce delays that cause the lag between prey and predator peaks to increase. This work explains and unifies empirical and theoretical studies on how predator-prey coevolution alters the dynamics of predator-prey systems and how those effects depend on the magnitudes of prey and predator standing genetic variation.
\end{abstract}

Key words: adaptive dynamics; coevolution; community dynamics; eco-evolutionary feedbacks; heritability; loop analysis; population dynamics; stability.

\section{INTRODUCTION}

Increased genetic variation within a species can alter the ecological dynamics and composition of communities. Increased genetic variation within a focal species can promote coexistence with their competitors (Lankau and Strauss 2007, Clark 2010), their exploiters (Imura et al. 2003, Coberly et al. 2009), or other species in the community (Crutsinger et al. 2006, Johnson et al. 2009, Utsumi 2015). Empirical studies have also shown that increased genetic variation can allow for evolution to alter the stability (Agashe 2009, Becks et al. 2010, Steiner and Masse 2013, Hiltunen and Becks 2014) and population dynamics (Yoshida et al. 2003, 2007, Nachappa et al. 2011, Hiltunen et al. 2014) of communities. However, altered genetic variation does not always lead to large changes in community composition (Fridley and Grime 2010, Ingram et al. 2011). In addition, changes in population dynamics may only occur if the magnitude of genetic variation within a species is sufficiently large (Becks et al. 2010). In other words, evolution can have important effects on community-level properties, but the magnitudes of those effects may depend on the levels

Manuscript received 2 January 2018; revised 15 February 2018; accepted 21 March 2018. Corresponding Editor: Bruce E. Kendall.

${ }^{1}$ E-mail: michael.cortez@usu.edu of standing genetic variation within the evolving species. This body of empirical work motivates questions about how evolution and standing genetic variation influence the dynamics of ecological communities (Bolnick et al. 2011, Shefferson and Salguero-Gómez 2015).

As a step toward answering questions about when and why evolution alters community-level dynamics, this study explores how evolution and altered genetic variation affect the population-level dynamics and stability of predator-prey systems. Predator-prey systems are an important class of systems to study because previous empirical studies have shown that evolution in prey and/or predators can alter the cyclic dynamics of predator-prey systems and drive cycles that strongly contrast with predictions from classical ecological theory without evolution (Fig. 1). In particular, ecological theory predicts predator-prey cycles where prey peaks precede predator peaks by up to one quarter of the cycle period (Fig. 1A, B; Rosenzweig and MacArthur 1963, Bulmer 1975). In contrast, in some empirical systems, the evolution of prey defense has been observed to drive oscillations where prey peaks precede predator peaks by one-half period (Yoshida et al. 2003); these are known as antiphase cycles (Fig. 1C, D). Prey evolution has also been observed to drive an extreme form of antiphase oscillations, called cryptic cycles (Fig. 1E, F), wherein the predator population exhibits large fluctuations in abundance while prey abundance is 
essentially constant (Yoshida et al. 2007). Predator-prey coevolution can also drive antiphase oscillations (Frickel et al. 2016, Haafke et al. 2016). In addition, cycles where peaks in prey density follow peaks in predator density have been observed in empirical systems (Fig. 1G, H); these are known as clockwise cycles due to their clockwise orientation in the predator-prey phase plane $($ Fig. $1 \mathrm{H})$, and are predicted to be caused by predator-prey coevolution (Cortez and Weitz 2014, Cortez 2015). Taken together, this range of cycle characteristics shows that evolution can qualitatively alter the dynamics of empirical predator-prey systems.

A second reason why predator-prey systems are important to study is that previous empirical studies have shown that the effects of evolution on cyclic dynamics and stability depend on the amounts of standing prey and predator genetic variation. For example, in one rotifer-algae system (Becks et al. 2010), the system converged to a steady state when prey genetic variation was low, but increased genetic variation was destabilizing and caused antiphase cycles. In contrast, in another rotifer-algae system (Steiner and Masse 2013) increased prey genetic variation was stabilizing. Importantly, in both systems, prey defense evolved over time in both the low and high variation treatments. Thus, the observed changes in stability were driven by the altered amounts of prey genetic variation, not the presence or absence of prey evolution. Increased predator genetic variation has also been observed to destabilize a ciliatebacteria system (Hiltunen and Becks 2014). Altogether, this body of empirical work shows that evolution in one or both species can alter the dynamics of predator-prey systems, and that those effects may depend on the
A

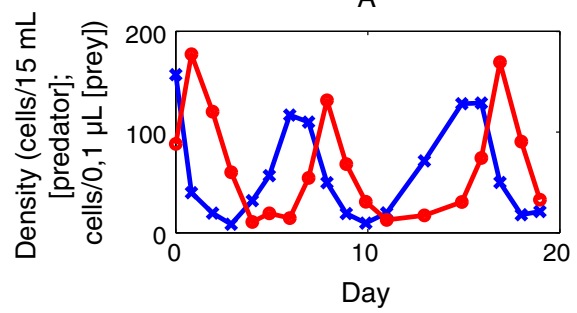

C

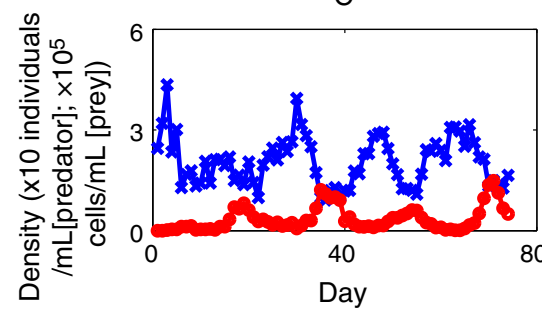

E

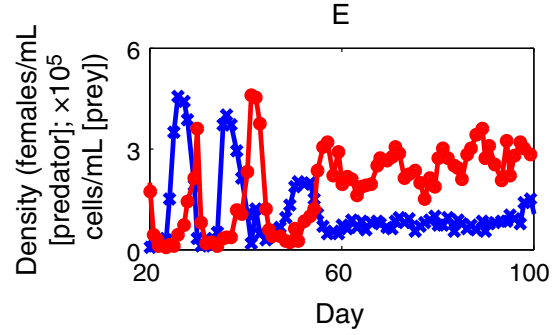

G

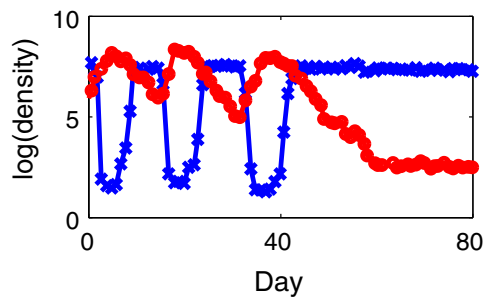

$\mathrm{B}$

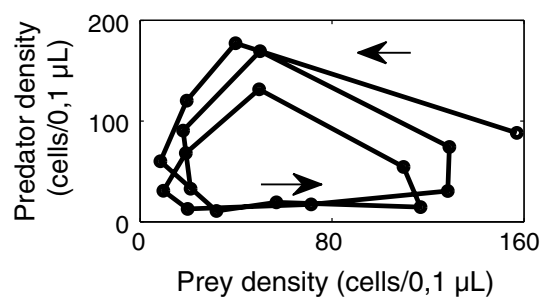

D

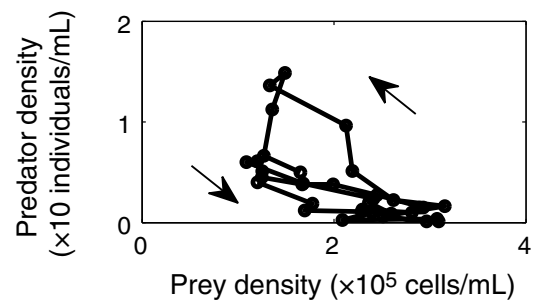

$\mathrm{F}$

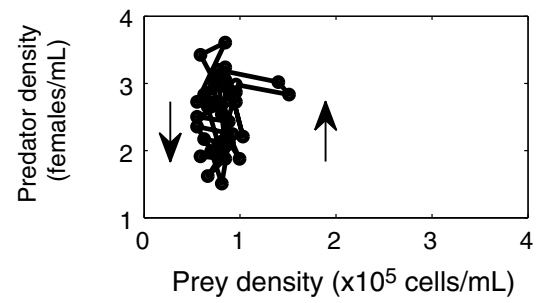

$\mathrm{H}$

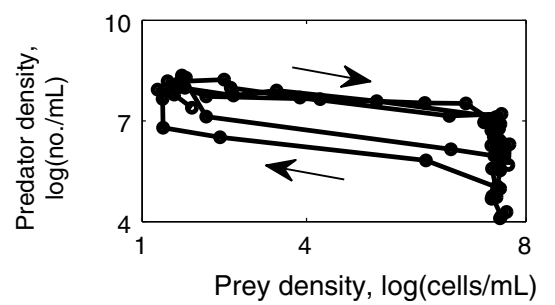

FIG. 1. Empirical examples of different types of predator-prey cycles. (A, B) Counterclockwise cycles of Paramecium aurelia (cells/ $0.1 \mu \mathrm{L}$ ) and Saccharomyces exiguus (cells/15 mL) from Gause (1935). (C, D) Antiphase cycles of Brachionus calyciflorus (10 individual/mL) and Chlamydomonas reinhardtii $\left(10^{5}\right.$ cells $/ \mathrm{mL}$ ) from Becks et al. (2010). (E, F) Cryptic cycles of Brachionus calyciflorus (females/mL) and Chlorella vulgaris $\left(10^{5}\right.$ cells $\left./ \mathrm{mL}\right)$ from Yoshida et al. (2007). (G, H) Clockwise cycles of LPP-1 cyanophage (number $/ \mathrm{mL}$ ) and Plectonema boryanum (cells $/ \mathrm{mL}$ ) from Cannon et al. (1976). The left column shows time series of prey (blue + ) and predator (red circles) densities. The right column shows the cycles plotted in the predator-prey phase plane; arrows denote the flow of time. For clarity, only the second halves of the time series are shown in panels D and $\mathrm{F}$. 
amounts of standing genetic variation in the predator and prey populations.

The existing body of eco-evolutionary theory helps to explain some of the above empirical patterns. One part of the theory explores and identifies the biological conditions under which prey evolution (Abrams and Matsuda 1997b, Jones and Ellner 2007, Yoshida et al. 2007, Cortez and Ellner 2010), predator evolution (Abrams 1992, Cortez and Ellner 2010, Yamamichi et al. 2015), and coevolution (Abrams and Matsuda 1997a, Jones et al. 2009, Cortez and Weitz 2014, Cortez 2015) alter the stability and cyclic dynamics of predator-prey systems. Importantly, that theory assumes that the amounts of genetic variation in all evolving species are sufficiently high for evolution to alter the dynamics of the system. A second complementary part of the theory explores how varying the amount of genetic variation in one or both species affects the dynamics and stability of models including prey evolution (Cortez 2016), predator evolution (Abrams 1992, Cortez and Patel 2017), or coevolution (Saloniemi 1993, Mougi and Iwasa 2011, Mougi 2012a, Tien and Ellner 2012, van Velzen and Gaedke 2017).

While this theory highlights how evolution and altered genetic variation in one or both species influence population-level dynamics, it is limited in three ways. First, due to the specificity of the models that have been studied, previous models of coevolution have been lacking in generality. Second, little attention has been paid to how the results of studies on single evolving species emerge from coevolutionary models. Intuition suggests that single-species evolutionary theories are special cases of coevolutionary theory, however it is not currently clear how to fit those bodies of theory together. Third, current theory does not provide a way to identify general mechanisms that organize and unify phenomena observed across systems. Of particular interest is the role that ecological, evolutionary, and eco-evolutionary feedbacks play in driving observed dynamics. For example, for destabilization to occur with increased genetic variation in the rotifer-algae (Becks et al. 2010) and ciliate-bacteria (Hiltunen and Becks 2014) systems from above, there must be some positive feedback between the ecological and/or evolutionary processes. In addition, feedbacks between ecological and evolutionary processes must play some part in differentiating between coevolution-driven antiphase cycles (Frickel et al. 2016, Haafke et al. 2016) and clockwise cycles (Cortez and Weitz 2014). However, the current theory does not provide insight about which feedbacks are responsible for driving these patterns. Thus, we cannot currently make general predictions about which processes or mechanisms are driving the different phenomena observed across systems.

This paper explores how altered genetic variation in one or both species influences the stability and population dynamics of predator-prey systems. The three main contributions of this study are the following. First, using a more coarse-grained, but mathematically equivalent, version of Levins' loop analysis (Levins 1974) and the phase lag theory in Ellner and Becks (2011), this study identifies how ecological, evolutionary, and eco-evolutionary feedbacks and indirect effects alter the stability and cyclic dynamics of predator-prey systems. In particular, I focus on how the magnitudes of standing prey and predator genetic variation influence the relative strengths of the different feedbacks and indirect effects. Second, the theory unifies the above body of theory on how evolution and varied genetic variation in one or both species alters predator-prey dynamics. Third, I identify the specific biological mechanisms and conditions that define when positive feedbacks and indirect effects will alter populationlevel dynamics. This, in turn, helps explain why qualitatively different types of predator-prey cycles occur across empirical systems.

\section{Models And Definitions}

\section{Eco-coevolutionary predator-prey model}

Throughout, I focus on an eco-coevolutionary predatorprey model that describes how the total prey density $(x)$, total predator density $(y)$, mean prey defense $(\alpha)$, and mean predator offense ( $\beta$ ) change over time. In the model, higher prey defense (large $\alpha$ ) comes at the cost of decreased reproductive output, e.g., increased defense against consumption by rotifers comes at the cost of decreased intraspecific competitive ability in algae (Yoshida et al. 2003). Higher predator offense (large $\beta$ ) comes at the cost of increased mortality, e.g., resistance to newt toxicity in garter snakes comes at the cost of decreased survival via reduced speed (Brodie and Brodie 1999). The model was studied previously in the fast evolution limit, where rates of evolution were much faster than rates of change in population densities (Cortez and Weitz 2014, Cortez 2015). In this study, I explore the dynamics when rates of evolutionary change are slower, comparable to, or faster than rates of ecological change.

The model equations are

$$
\begin{gathered}
\frac{d x}{d t}=\overbrace{x f\left(x, \alpha, \alpha_{i}\right)}^{\text {reproduction }}-\left.\overbrace{x g\left(x, y, \alpha, \alpha_{i}, \beta\right)}^{\text {predation }}\right|_{\alpha_{i}=\alpha} \\
\frac{d y}{d t}=\underbrace{y h\left(x, y, \alpha, \beta, \beta_{i}\right)}_{\text {reproduction }}-\left.\underbrace{y d\left(y, \beta, \beta_{i}\right)}_{\text {mortality }}\right|_{\beta_{i}=\beta} \\
\frac{d \alpha}{d t}=V_{x} \frac{\partial}{\left.\underbrace{\frac{\partial}{x}}_{\text {fitness gradient }}\left[\frac{1}{x} \frac{d x}{d t}\right]\right|_{\alpha_{i}=\alpha}} \\
\frac{d \beta}{d t}=V_{y} \overbrace{\left.\frac{\partial}{\partial \beta_{i}}\left[\frac{1}{y} \frac{d y}{d t}\right]\right|_{\beta_{i}=\beta}}^{\text {fitness gradient }}
\end{gathered}
$$

Eqs. $1 \mathrm{a}$ and $1 \mathrm{~b}$ form the ecological component of the model and describe how the prey and predator populations increase and decrease due to reproduction and mortality. Specifically, $f$ is the net per capita reproduction rate of the 
prey in the absence of predators, $x g$ is the predation rate, $y h$ is the composition of the predation rate and predator-toprey conversion, and $d$ is the predator per capita mortality rate. Importantly, the ecological dynamics of the model depend on the mean levels of prey defense and predator offense. The variables $\alpha_{i}$ and $\beta_{i}$ denote the trait values of an individual prey and predator, which are evaluated at the mean trait values because the population dynamics of the model depend on the mean trait values; see the next section for additional information.

Eqs. 1c and 1d form the evolutionary component of the model. The equations describing the evolution of prey defense and predator offense are are derived from quantitative genetics theory (Lande 1976, 1982, Iwasa et al. 1991, Taper and Case 1992) adapted to continuous time models (Abrams et al. 1993). In those equations, the rates of evolution are proportional to the additive genetic variation in the populations $\left(V_{x}, V_{y}\right)$ and the individual fitness gradients (see the next section for more details). The direction of selection is determined by the fitness gradient. The speed of evolution is determined by both the magnitude of genetic variation and the steepness of the fitness gradient.

In this study, I explore how the speed of evolution affects the stability and cyclic dynamics of model (1). To simplify the presentation, I focus on interpreting the speed of evolution in terms of the magnitude of additive genetic variation. In particular, rates of evolutionary change are slower than rates of ecological change when genetic variation is low ( $V_{x} \ll 1, V_{y} \ll 1$ ), e.g., when evolution is mutation limited. Rates of evolutionary and ecological change are comparable when genetic variation is intermediate $\left(V_{x} \approx 1, V_{y} \approx 1\right)$. This occurs when selection is weak relative to mutation (or other processes maintaining genetic variation), or when genotypes are present (possibly at low densities) and evolution occurs as genotype frequencies change over time. Finally, rates of evolutionary change are faster than rates of ecological change when genetic variation is high $\left(V_{x} \gg 1, V_{y} \gg 1\right)$. The fast evolution limit is less likely to occur in natural systems, however it is a useful approximation for making inferences about eco-evolutionary dynamics (Cortez and Ellner 2010, Patel et al., 2018).

\section{Frequency-dependent vs. -independent selection in the model}

In model (1), the individual trait values for the prey $\left(\alpha_{i}\right)$ and predator $\left(\beta_{i}\right)$ are place-holding variables that allow for frequency-dependent selection. Because the prey and predator population-level dynamics depend on the mean trait values, the individual trait values are evaluated at the mean trait values in Eqs. 1a and 1b. However, because frequencydependent selection depends on individual fitness, which depends on an individual's trait value, the fitness gradients in the evolution Eqs. 1c and 1d involve derivatives taken with respect to the individual trait values.

To see how frequency-dependent vs. frequency-independent selection can arise in practice in the model, consider a prey population that grows logistically in the absence of predators and whose predator has as Type II functional response. The dynamics of the total prey population are

$$
\frac{d x}{d t}=r(\alpha) x\left(1-\frac{x}{K}\right)-\frac{a(\alpha, \beta) x y}{1+h a(\alpha, \beta) x}
$$

where $r(\alpha)$ is the trait-dependent maximum exponential growth rate of the prey, $K$ is the carrying capacity, $a$ is the trait-dependent predator-prey encounter rate, and $h$ is the predator handling time. Importantly, the dynamics of the total prey population depend on the mean levels of offense ( $\beta$ ) and defense $(\alpha)$. For the subpopulation of prey that have trait $\alpha_{i}$, their dynamics are $d x_{i} / d t=r\left(\alpha_{i}\right) x_{i}(1-x / K)-$ $a\left(\alpha_{i}, \beta\right) x_{i} y /[1+h a(\alpha, \beta) x]$, where $r\left(\alpha_{i}\right)$ and $a\left(\alpha_{i}, \beta\right)$ are the growth and encounter rates for individuals with trait $\alpha_{i}$. Importantly, the term $a\left(\alpha_{i}, \beta\right)$ in the numerator of the functional response depends on the individual trait value because it defines the encounter rate between individuals with trait $\alpha_{i}$ and predators. In contrast, the term $a(\alpha, \beta)$ in the denominator depends on the mean prey trait value $(\alpha)$ because the mean trait value defines the average predator grazing rate for the entire prey population. Consequently, when computing the individual fitness gradient, only the derivative of the term $a\left(\alpha_{i}, \beta\right)$ in the numerator of the functional response will be used. The equations for the frequency-dependent evolutionary dynamics of the prey population are

$$
\frac{d \alpha}{d t}=\left.V_{x} \frac{\partial}{\partial \alpha_{i}}\left[\frac{1}{x} \frac{d x}{d t}\right]\right|_{\alpha_{i}=\alpha}=V_{x}\left[r_{\alpha}(\alpha)\left(1-\frac{x}{K}\right)-\frac{a_{\alpha}(\alpha, \beta) y}{1+h a(\alpha, \beta) x}\right]
$$

where the subscript $\alpha$ denotes partial differentiation, i.e., $r_{\alpha}(\alpha)=\frac{\partial}{\partial \alpha} r(\alpha)$ and $a_{\alpha}(\alpha, \beta)=\frac{\partial}{\partial \alpha} a(\alpha, \beta)$.

In contrast, if selection is frequency independent then the evolution equation becomes

$$
\frac{d \alpha}{d t}=V_{x} \frac{\partial}{\partial \alpha}\left[\frac{1}{x} \frac{d x}{d t}\right]=V_{x}\left[r_{\alpha}(\alpha)\left(1-\frac{x}{K}\right)-\frac{a_{\alpha}(\alpha, \beta) y}{[1+h a(\alpha, \beta) x]^{2}}\right]
$$

There are two key differences between Eqs. 3 and 4. First, because selection is frequency independent in Eq. 4, the fitness gradient is defined by the derivative with respect to the mean trait value $(\alpha)$, not the individual trait value $\left(\alpha_{i}\right)$. Second, this causes the right hand sides of Eqs. 3 and 4 to differ. In particular, the squared denominator of the last term in Eq. 4 is due to the derivatives of the $a(\alpha, \beta)$ terms in the numerator and denominator of the functional response.

\section{The Jacobian matrix, subsystems, direct and indirect effects, and feedback loops}

My results about system stability and predator-prey phase lags are based on the Jacobian matrix. The Jacobian matrix determines whether small perturbations to an equilibrium decay (implying stability) or grow (implying instability). The Jacobian matrix is shown in Fig. 2B. The signs of the entries of the Jacobian matrix are determined by the ecological interactions between the species and the fitness effects of increased offense and defense. Definitions and signs of the Jacobian matrix entries are given in Table 1; see 
Appendix S1: Section S2 for more details. Components of the Jacobian matrix also define three other useful quantities: subsystems, direct and indirect effects, and feedback loops.

A subsystem describes the dynamics of 1,2 , or 3 variables when the other variables are fixed at their equilibrium values. One-dimensional subsystems describe the dynamics of a single variable when all other variables are fixed. For example, the prey evolutionary subsystem describes the prey evolutionary dynamics when the species' densities and the predator trait are fixed. Note that the dynamics of this subsystem correspond to the dynamics of Eq. 1c when $x, y$, and $\beta$ are fixed at their equilibrium values. Two-dimensional subsystems describe the dynamics of two variables when the other two variables are fixed at their equilibrium values. For example, the coevolutionary subsystem describes the coevolutionary dynamics of the system (Fig. 2C) when the prey and predator densities are fixed; the dynamics of this subsystem correspond to the dynamics of Eqs. 1c and 1d when the species densities are fixed at their equilibrium values. Threedimensional subsystems describe the dynamics of three variables when the fourth variable is fixed. For example, the three-dimensional eco-evolutionary subsystem in Fig. 2A corresponds to the dynamics of the species densities and the prey trait when the predator trait is fixed, i.e., Eqs. 1a-c when $\beta$ is fixed at its equilibrium value.

The stabilities of the subsystems are determined by submatrices of the Jacobian matrix. In general, for a given subsystem, the corresponding submatrix is made up of all entries of the Jacobian matrix that include only those variables. For one-dimensional subsystems, the corresponding submatrices are the diagonal entries of the Jacobian matrix. For example, the submatrix defining the stability of the prey evolutionary subsystem is just the 3,3-entry of the Jacobian matrix $\left(J_{33}\right)$. For two-dimensional subsystems, the $2 \times 2$ submatrices are made up of entries of the Jacobian matrix that only involve the two variables in that subsystem. For example, the coevolutionary subsystem where the traits change and the densities are fixed (Fig. 2C) corresponds to the $2 \times 2$ matrix in the bottom right corner of the Jacobian matrix (black box in the bottom right of Fig. 2B). For three-dimensional subsystems, the $3 \times 3$ submatrices are made up of entries of the Jacobian matrix that only involve the three variables in that subsystem. For example, the eco-evolutionary subsystem involving both species densities and the prey trait (Fig. 2A) corresponds to the $3 \times 3$ matrix in the top left corner of the Jacobian matrix (black box in the top left of Fig. 2B). In total, the Jacobian matrix defines the stability of the whole system and submatrices of the Jacobian matrix define the stabilities of subsystems.

Direct and indirect effects describe how changes in one variable directly or indirectly influence its own dynamics or the dynamics of other variables. In general, a single entry of the Jacobian matrix, $J_{i j}$, denotes the direct effect of variable $j$ on the dynamics of variable $i$. I depict direct effects between different variables using straight arrows $(\rightarrow)$ and direct effects of a variable on itself (self-effects) using circular arrows $(\circlearrowleft)$. For example, $J_{33}$ is the self-effect of the prey trait on its own dynamics $(\alpha \circlearrowleft)$ and $J_{13}$ is the direct effect of the prey trait on the prey population dynamics $(\alpha \rightarrow x)$. Indirect effects are defined by products of the off-diagonal Jacobian matrix entries. For example, $J_{34} J_{43}$ defines the indirect effect of the prey trait on its own dynamics mediated by the predator trait. This indirect effect can be depicted by a chain of straight arrows $(\alpha \rightarrow \beta \rightarrow \alpha)$, or more concisely as stacked arrows $(\alpha \rightleftarrows \beta)$. Here, $J_{43}$ defines the effect of the prey trait on the predator trait dynamics $(\alpha \rightarrow \beta)$ and $J_{34}$ defines the effect of the predator trait on the prey trait dynamics $(\alpha \leftarrow \beta)$. In general, $J_{i j} J_{j l}$ is the indirect effect of variable $l$ on the dynamics of variable $i$ mediated by a change in variable $j$ (depicted as $l \rightarrow j \rightarrow i$ ). Similarly, $J_{i j} J_{j k} J_{k l}$ is the indirect effect of variable $l$ on the dynamics of variable $i$ mediated by changes in variables $j$ and $k$ (depicted as $l \rightarrow k \rightarrow j \rightarrow i$ ).

Feedback loops describe the direct or indirect effects a variable has on its own dynamics (Levins 1974). Direct feedback loops are the direct effects of a variable on its own dynamics; they are represented by the diagonal entries of the Jacobian matrix. For example, the direct effect of the prey trait on its own dynamics is defined by the 3,3-entry of the Jacobian matrix $\left(J_{33}\right)$. Indirect feedback loops are the indirect effects of a variable on its own dynamics; they are represented by products of off-diagonal entries of the Jacobian matrix, i.e., products of indirect effects. For example, the indirect effect of the prey trait on its own dynamics mediated by the predator trait is represented by $J_{34} J_{43}$. When three variables are involved, there are two possible indirect
A

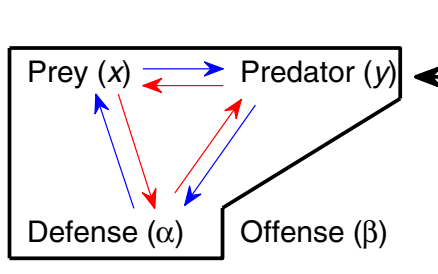

Prey Eco-Evo Subsystem
B
C

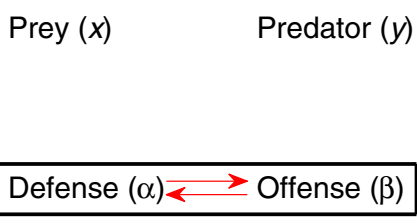

FIG. 2. The Jacobian matrix determines the stability of subsystems via submatrices and direct and indirect feedbacks via its entries. (B) Jacobian matrix for model (1); see Table 1 for descriptions of terms. (A) The stability of the three-dimensional prey eco-evolutionary subsystem (enclosed variables) is determined by the upper left $3 \times 3$ submatrix of the Jacobian matrix (upper left box). The red counterclockwise and blue clockwise arrows and corresponding entries in the submatrix denote the two indirect eco-evolutionary feedback loops that involve the prey trait and both species densities. (C) The stability of the coevolutionary subsystem (enclosed variables) is determined by the bottom right $2 \times 2$ submatrix of the Jacobian matrix (lower right box). The red arrows and corresponding entries in the submatrix denote the indirect coevolutionary feedback loop. 
TABLE 1. Interpretation and signs of Jacobian matrix entries evaluated at equilibrium.

\begin{tabular}{|c|c|c|}
\hline Value† & Description of effect & Sign: \\
\hline$J_{11}=x f_{x}-x g_{x}$ & $\begin{array}{l}\text { Effect of prey density on prey } \\
\text { growth rate }\end{array}$ & \pm \\
\hline$J_{12}=-x g_{y}$ & $\begin{array}{l}\text { Increased predator density decreases } \\
\text { prey growth rate }\end{array}$ & - \\
\hline$J_{13}=x f_{\alpha}-x g_{\alpha}$ & $\begin{array}{l}\text { Effect of mean prey defense on } \\
\text { individual fitness }\end{array}$ & \pm \\
\hline$J_{14}=-x g_{\beta}$ & $\begin{array}{l}\text { Increased offense decreases prey } \\
\text { growth rate }\end{array}$ & - \\
\hline$J_{21}=y h_{x}$ & $\begin{array}{l}\text { Increased prey density increases } \\
\text { predator growth rate }\end{array}$ & + \\
\hline$J_{22}=y h_{y}-y d_{y}$ & $\begin{array}{l}\text { Intraspecific competition decreases } \\
\text { predator growth rate }\end{array}$ & $-/ 0$ \\
\hline$J_{23}=y h_{\alpha}$ & $\begin{array}{l}\text { Increased defense decreases } \\
\text { predator growth rate }\end{array}$ & - \\
\hline$J_{24}=y h_{\beta}-y h_{\beta}$ & $\begin{array}{l}\text { Effect of mean predator offense on } \\
\text { individual fitness }\end{array}$ & \pm \\
\hline$J_{31}=V_{x}\left(f_{x \alpha_{i}}-g_{x \alpha_{i}}\right)$ & $\begin{array}{l}\text { Effect of increased prey density on } \\
\text { selection for defense }\end{array}$ & \pm \\
\hline$J_{32}=-V_{x} g_{y \alpha_{i}}$ & $\begin{array}{l}\text { Increased predator density increases } \\
\text { selection for defense }\end{array}$ & + \\
\hline $\begin{array}{l}J_{33}=V_{x}\left(f_{\alpha_{i} \alpha_{i}}-g_{\alpha_{i} \alpha_{i}}\right. \\
\left.\quad+f_{\alpha_{i} \alpha}-g_{\alpha_{i} \alpha}\right)\end{array}$ & $\begin{array}{l}\text { Stabilizing }\left(J_{33}<0\right) \text { or disruptive } \\
\left(J_{33}>0\right) \text { selection }\end{array}$ & \pm \\
\hline$J_{34}=-V_{x} g_{\beta_{\alpha_{i}}}$ & $\begin{array}{l}\text { Effect of increased offense on } \\
\text { selection for defense }\end{array}$ & \pm \\
\hline$J_{41}=V_{y} h_{x \beta_{i}}$ & $\begin{array}{l}\text { Effect of increased prey density on } \\
\text { selection for offense }\end{array}$ & \pm \\
\hline$J_{42}=V_{y}\left(h_{y \beta_{i}}-d_{y \beta_{i}}\right)$ & $\begin{array}{l}\text { Increased predator density decreases } \\
\text { selection for offense }\end{array}$ & $-/ 0$ \\
\hline$J_{43}=V_{y} h_{\alpha \beta_{i}}$ & $\begin{array}{l}\text { Effect of increased defense on } \\
\text { selection for offense }\end{array}$ & \pm \\
\hline $\begin{array}{l}J_{44}=V_{y}\left(h_{\beta_{i} \beta_{j}}-d_{\beta_{i} \beta_{i}}\right. \\
\left.+h_{\beta_{i} \beta}-d_{\beta_{i} \beta}\right)\end{array}$ & $\begin{array}{l}\text { Stabilizing }\left(J_{44}<0\right) \text { or disruptive } \\
\left(J_{44}>0\right) \text { selection }\end{array}$ & \pm \\
\hline
\end{tabular}

$\dagger$ Subscripts denote partial derivatives, e.g., $\partial f / \partial x=f_{x}$. The values of $J_{11}, J_{22}, J_{13}$, and $J_{24}$ are simplified after taking into account that the Jacobian matrix is evaluated at a coexistence equilibrium; see Appendix S1: Section S2 for details.

tValues that can have either sign are denoted by \pm . Values that can be negative or zero are denoted $-/ 0$.

feedback loops. For example, $J_{31} J_{12} J_{23}$ and $J_{32} J_{21} J_{13}$ represent the two indirect feedback loops of the prey trait that are mediated by the prey and predator densities.

The connections between subsystems, feedback loops, and direct and indirect effects are the following. Direct and indirect effects are components of direct and indirect feedback loops and define whether the feedback loops are stabilizing (negative feedback loops) or destabilizing (positive feedback loops). Feedback loops in turn determine whether subsystems are stable or unstable. Subsystems then determine whether the full system is stable or unstable. Thus, the stability and dynamics of the eco-evolutionary predator-prey model (1) can be interpreted in terms of the effects of subsystems (the dynamics of subsets of variables), feedback loops (the direct and indirect effects of variables on their own dynamics), and direct effects (the effects of changes in one variable on the dynamics of another variable).

\section{Assumptions and generality of model and results}

This section addresses the generality and assumptions underlying the model and the results. Readers not interested in the details can skip this section without loss of comprehension. Additional mathematical details about the model are provided in Appendix S1.

I use the general functions in model (1) instead of specific functional forms in order to develop general theory that can be applied to many different systems. However, a number of specific assumptions are built into model (1), both to match the biological conditions typical of predator-prey systems and to simplify the model. First, predation rates increase with predator and prey densities. This assumption is satisfied by typical functional forms used in eco-evolutionary predator-prey models, e.g., Type I, II, or III functional responses. Second, the general functions are assumed to satisfy the trade-off between prey defense and reproduction and the trade-off between predator offense and mortality. Finally, the model also assumes that the prey and predator genetic variances $\left(V_{x}, V_{y}\right)$ are constant.

Importantly, while the model is assumed to satisfy these specific conditions, because of the level of generality of the model and the methods used in this study, the results of this study apply to any model that has the same general structure as model (1). Here, a model has the same general structure if (1) the population dynamics of each species are described by a single equation (i.e., there is no stage structure in either population) and (2) the evolutionary dynamics for each species are defined by a single equation that describes how a single characteristic of the trait distribution (e.g., the mean trait value) changes over time. Any model that fits these criteria will have a Jacobian matrix with the same structure as the Jacobian matrix for model (1). Therefore, because the methods used in this study only depend on the magnitudes and signs of the Jacobian matrix entries, and not the particular model components or parameters used to compute those entries, the results of this study will apply to any model satisfying the above criteria with only minor differences in interpretation. Eco-evolutionary models of particular interest include Saloniemi (1993), which assumed stabilizing selection in both species; Tien and Ellner (2012), where the rate of prey evolution was proportional to the standing genetic variation, the fitness gradient, and the mean prey trait value; and models whose evolution equations are derived from the theory of Adaptive Dynamics (Dieckmann et al. 1995, Marrow et al. 1996, Geritz et al. 1998). Below I address specific assumptions and aspects of the model.

First, the assumed trade-offs between prey defense and reproduction and predator offense and mortality are likely to be common, but other trade-offs are possible (e.g., a tradeoff between prey defense and mortality due to other causes or a trade-off between predator attack rate and conversion efficiency; Abrams 1986). In addition, previous studies have focused on two kinds of traits: unidirectional traits and bidirectional traits (Abrams 2000). This study focuses on unidirectional traits, where higher offense results in increased predation of all prey types and higher defense results in decreased predation from all predator types; see Tien and Ellner (2012) and Mougi and Iwasa (2011) for other examples. Predators and prey may instead have bidirectional traits where the predator trait needs to match the prey trait in order to achieve a high capture rate; see Mougi (2012a) for an example. In all cases, these differences in assumptions about the traits only influence some of the signs of the Jacobian matrix entries (e.g., assumptions about the prey 
trait and trade-off affect $J_{13}$ and $J_{31}$ ). Thus, the theory developed in this study applies directly after accounting for the altered signs.

Second, while model (1) assumes constant standing predator and prey genetic variation, in general genetic variation changes over time. Because the methods in this study focus on the stability of equilibrium points, all of the stability results apply to models where genetic variation changes over time, provided that the values of $V_{x}$ and $V_{y}$ in model (1) are set equal to the equilibrium values of the genetic variances in the model with variable genetic variation (Cortez 2016). One special case of interest is Adaptive Dynamics models (e.g., Marrow et al. 1996), where $V_{i}$ is replaced by the product of the mutation rate, the mutation step variances, and the (ecological equilibrium) population density. All of my stability results for slow evolution apply to the eco-evolutionary equilibrium points of those models after computing the values for $V_{x}$ and $V_{y}$. The results for predator-prey phase lags also hold for varying genetic variation, so long as the changes in genetic variation are small. Whether the results hold for larger changes in genetic variation is model specific because it depends on nonlocal properties of the model; see How genetic variation alters predator-prey phase lags and Appendix S3: Section S1 for more details.

Third, I focus on interpreting the rate of evolution in terms of the magnitude of genetic variation. However, the rate of evolution is also determined, in part, by the steepness of the fitness gradient. All of my results can be interpreted in terms of the steepness of the fitness gradient. Specifically, the slow evolution results apply to systems with low genetic variation and/or shallow fitness gradients and the fast evolution results apply to systems with high genetic variation and/ or steep fitness gradients. Note that because additive genetic variation is the product of phenotypic variation and narrow-sense heritability, changes in genetic variation can be interpreted as changes in one or both quantities. While changes in either quantity have the same effect in model (1), this may not hold for other models where the predation rates depend on the levels of prey and predator phenotypic variation; see Schreiber et al. (2011) and Patel and Schreiber (2015) for examples. Nonetheless, my results apply to those models so long as changes in genetic variation only reflect changes in heritability (for fixed levels of phenotypic variation).

Note that when presenting results, I discuss and contrast cases where genetic variation is high, intermediate, and low. Mathematically, high genetic variation means evolution is an order of magnitude faster than ecology $\left(V_{x} \gg 1\right.$, $\left.V_{y} \gg 1\right)$, intermediate variation means rates of evolution and ecological are comparable $\left(V_{x} \approx 1, V_{x} \approx 1\right)$, and low genetic variation means evolution is an order of magnitude slower than ecology $\left(V_{x} \ll 1, V_{y} \ll 1\right)$. However, in practice, high and low genetic variation do not require a separation of time scales between the ecological and evolutionary processes. Moreover, while specific ranges are model dependent, phrases like "sufficiently low" or "sufficiently high" genetic variation mean that there exists a threshold value under or over which a phenomenon occurs. For example, sufficiently low (high) prey genetic variation could mean $V_{x} \leq 0.9$ $\left(V_{x} \geq 0.5\right)$ for one-one model and $V_{x} \leq 0.1 \quad\left(V_{x} \geq 10\right)$ for another.
Finally, gradient dynamics models, like model (1), are a first approximation to many kinds of evolutionary models, including systems with discrete traits (e.g., clonal systems; Abrams and Matsuda 1997b, Cortez and Weitz 2014) or continuous traits (Abrams and Matsuda 1997b) undergoing stabilizing or disruptive selection (Turelli and Barton 1994). In addition, their simplicity makes them analytically tractable and allows one to study evolutionary dynamics at the phenotypic level without specifying gene-level processes. This makes gradient dynamic models a good starting point for studying eco-evolutionary dynamics. But, because they do not specify gene-level processes, they may not capture all possible dynamics (e.g., see Levin and Udovic 1977, Doebeli 1997, Yamamichi and Ellner 2016).

\section{RESULTS}

\section{System stability via Levins' loop analysis}

To determine how the ecological, evolutionary, and ecoevolutionary subsystems and feedback loops influence the stability of the whole system, I use a more coarse-grained, but mathematically equivalent, version of Levins' loop analysis (Levins 1974). My approach is based on the Routh-Hurwitz stability criterion (e.g., Edelstein-Keshet 1989, Gantmacher 1998). Briefly, the characteristic polynomial of the Jacobian matrix is

$$
p(\lambda)=\lambda^{4}+a_{1} \lambda^{3}+a_{2} \lambda^{2}+a_{3} \lambda+a_{4}
$$

The coefficients of the characteristic polynomial are used to construct the sequence

$$
\left\{1, a_{1}, a_{1}\left(a_{1} a_{2}-a_{3}\right), a_{3}-\frac{a_{1}^{2} a_{4}}{a_{1} a_{2}-a_{3}}, a_{4}\right\} .
$$

An equilibrium point of model (1) is stable only when all entries in the sequence are positive; if any of the entries are negative then the system is unstable. Note that for this model, instability of a coexistence equilibrium implies there are predator-prey cycles; see Appendix S1: Section S2 for details.

The coefficients in the characteristic polynomial decompose into terms representing the stabilities of different subsystems,

$$
\begin{aligned}
a_{1}= & -\overbrace{\left(M_{x}+M_{y}\right)}^{1 \mathrm{D} \text { Eco }}-\overbrace{V_{x} M_{\alpha}}^{\text {Prey evo }}-\overbrace{V_{y} M_{\beta}}^{\text {Predator evo }} \\
a_{2}= & \overbrace{\left|M_{x y}\right|}^{\text {Eco }}+\overbrace{V_{x}\left(\left|M_{x \alpha}\right|+\left|M_{y \alpha}\right|\right)}^{\text {D P Prey eco-evo }}+\overbrace{V_{y}\left(\left|M_{x \beta}\right|+\left|M_{y \beta}\right|\right)}^{2 \mathrm{D} \text { Predator eco-evo }} \\
& +\overbrace{V_{x} V_{y}\left|M_{\alpha \beta}\right|}^{\text {Coevo }}, \\
a_{3}= & -\overbrace{V_{x}\left|M_{x y \alpha}\right|}^{3 \mathrm{D} \text { Prey eco-evo }}-3 \mathrm{D} \text { Predator eco-evo } \\
& -\overbrace{V_{x} V_{y}\left(\left|M_{x \alpha \beta}\right|+\left|M_{x y \beta}\right|\right.}^{\text {Eco-coevo }}+ \\
a_{4}= & |J| .
\end{aligned}
$$


Here, $M_{i}, M_{i j}$, and $M_{i j k}$ are submatrices of the Jacobian matrix evaluated at $V_{x}=V_{y}=1$, where the indices denote the corresponding subsystems; see Table 2 for definitions. The vertical bars denote determinants, which determine if a subsystem is consistent with stability. For example, the $2 \times 2$ matrix for the coevolutionary subsystem in Fig. $2 \mathrm{C}$ is $M_{\alpha \beta}$. The term $V_{x} V_{y}\left|M_{\alpha \beta}\right|$ in the equation for coefficient $a_{2}$ represents the stability of that subsystem. Similarly, the $3 \times 3$ matrix for the eco-evolutionary subsystem in Fig. 2A is $M_{x y \alpha}$. The term $V_{x}\left|M_{x y \alpha}\right|$ in the equation for coefficient $a_{3}$ represents the stability of that subsystem. In general, the signs of $M_{i}$ and $\left|M_{i j k}\right|$ are consistent with stability when they are negative and the signs of $\left|M_{i j}\right|$ are consistent with stability when they are positive. Note that satisfying one of the conditions does not imply a subsystem is stable; the subsystem can exhibit cyclic dynamics. A subsystem that does not satisfy the conditions is unstable and satisfies the subsystem instability condition (sensu Cortez and Abrams 2016).

The biological interpretation of Eq. 7 is the following. Coefficient $a_{1}$ is the sum of the contributions of all onedimensional subsystems to the stability of the whole system. It accounts for the stabilities of the individual ecological and evolutionary processes for each species. Coefficient $a_{2}$ is the sum of the contributions of all two-dimensional subsystems to the stability of the whole system. It accounts for the stabilities of subsystems comprised of pairs of variables. Coefficient $a_{3}$ is the sum of the contributions of all threedimensional subsystems to the stability of the whole system. It accounts for the stabilities of subsystems comprised of triples of variables. In total, Eq. 7 shows that the stability of each subsystem influences the stability of the whole system through the $a_{i}$ coefficients. Thus, stability or instability of a particular subsystem can stabilize or destabilize the whole system, respectively. The next section addresses when particular subsystems have a strong influence on the stability of the whole system.

\section{Genetic variation determines which subsystems affect the stability of the whole system}

In this section, I show how the amounts of genetic variation determine which subsystems influence the stability of the whole system. The key thing to note is that the

TABLE 2. Names and notation for subsystems and submatrices of model (1).

\begin{tabular}{ll}
\hline \hline Subsystem & Submatrix \\
\hline One dimensional & \\
Ecological & $M_{x}, M_{y}$ \\
Prey evolutionary & $M_{\alpha}$ \\
Predator evolutionary & $M_{\beta}$ \\
Two dimensional & \\
Ecological & $M_{x y}$ \\
Prey eco-evolutionary & $M_{x \alpha}, M_{y \alpha}$ \\
Predator eco-evolutionary & $M_{x \beta}, M_{y \beta}$ \\
Coevolutionary & $M_{\alpha \beta}$ \\
Three dimensional & \\
Prey eco-evolutionary & $M_{x y \alpha}$ \\
Predator eco-evolutionary & $M_{x y \beta}$ \\
Eco-coevolutionary & $M_{x \alpha \beta}, M_{y \alpha \beta}$ \\
\hline
\end{tabular}

subsystem stability terms in Eq. 7 differentially depend on the amounts of prey $\left(V_{x}\right)$ and predator $\left(V_{y}\right)$ genetic variation. Specifically, the ecological subsystem terms are independent of the genetic variances, the evolutionary and ecoevolutionary subsystem terms depend only on the genetic variance of one species, and the coevolutionary and eco-coevolutionary subsystem terms depend on the genetic variances of both species. This means that the relative importance of those terms depends on the magnitudes of the prey and predator genetic variances. Intuitively, subsystems involving the prey (predator) trait have large effects on the stability of whole system when prey (predator) genetic variation is high and small effects when prey (predator) genetic variation is low.

The results are summarized in Fig. 3; mathematical details are given in Appendix S2: Section S2. When prey and predator genetic variation are low $\left(V_{x}, V_{y}\right.$ small; bottom left of Fig. 3), the stability of the whole system is determined by the stabilities of the one- and two-dimensional ecological subsystems and the three-dimensional eco-evolutionary subsystems. Intuitively, the ecological subsystems have relatively large effects because the effects of the evolutionary subsystems are weak when genetic variation is low. The eco-evolutionary subsystems also have large effects because they define how the slow evolutionary dynamics are influenced by the faster ecological dynamics.

When genetic variation is high in the prey and low in the predator ( $V_{x}$ large and $V_{y}$ small; bottom right of Fig. 3 ), the stability of the whole system is determined by the stabilities of the one-dimensional prey evolutionary subsystem and two- and three-dimensional eco-evolutionary subsystems that only involve the prey trait. Intuitively, the prey evolutionary subsystem has a large effect because increased genetic variation strengthens the effects of that subsystem. The eco-evolutionary subsystems involving the prey trait also have large effects because they define how the ecological dynamics of the prey and predator populations are influenced by the faster evolutionary dynamics of the prey. Following the same intuition, when genetic variation is low in the prey and high in the predator ( $V_{x}$ small and $V_{y}$ large; top left of Fig. 3), the stability of the whole system is determined by the stabilities of the one-dimensional predator evolutionary subsystem and two- and three-dimensional eco-evolutionary subsystems that only involve the predator trait.

Finally, when prey and predator genetic variation are both high ( $V_{x}, V_{y}$ large; top right of Fig. 3), the stability of the whole system is determined by the stabilities of the onedimensional prey and predator evolutionary subsystems, the two-dimensional coevolutionary subsystem and the threedimensional eco-coevolutionary subsystems. Intuitively, the effects of the single-trait evolutionary and coevolutionary subsystems are large because high genetic variation in one or both species strengthens the effects of those subsystems. The eco-coevolutionary subsystems also have large effects because they define how the ecological dynamics of the system are influenced by the faster coevolutionary dynamics.

Note that if prey genetic variation is intermediate $\left(V_{x} \approx 1\right)$, then the stability of the whole system is determined by the subsystems listed for both high and low prey genetic variation. For example, when prey genetic variation 


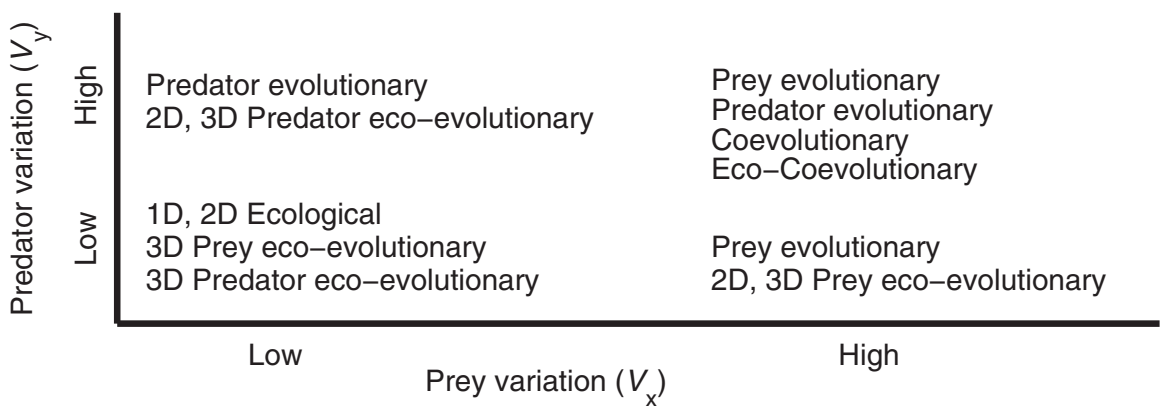

FIG. 3. Genetic variation determines which subsystems influence the stability of the whole system. See Table 2 for definitions of subsystems. If genetic variation in each species is either high or low, then the stability of the whole system is influenced by the subsystems in the corresponding quadrant. If genetic variation is intermediate for a species, then all subsystems listed for low and high genetic variation of that species influence the stability of the whole system. If genetic variation is intermediate for both species, all subsystems influence the stability of the system.

is intermediate and predator genetic variation is low (bottom middle of Fig. 3), system stability is determined by the ecological subsystems and all evolutionary and eco-evolutionary subsystems that only involve the prey trait. The same point applies to systems where predator genetic variation is intermediate. If prey and predator genetic variation are both intermediate $\left(V_{x} \approx 1, V_{y} \approx 1\right.$; center of Fig. 3), then all ecological, evolutionary, and eco-evolutionary subsystems affect the stability of the system.

To illustrate how this theory can be used to identify the underlying causes for instability of the whole system, consider the two examples in Fig. 4A, B; model equations are given in Appendix S5. Fig. 4A shows the stability for a model where a one-dimensional ecological subsystem $\left(M_{x}>0\right)$ and a two-dimensional prey eco-evolutionary subsystem $\left(\left|M_{x \alpha}\right|<0\right)$ are unstable. Hence, that system is predicted to be unstable when genetic variation is low in both species (bottom left) and when genetic variation is sufficiently low in the predator and sufficiently high in the prey (bottom right). Fig. 4B shows an example where a twodimensional eco-evolutionary subsystem involving just the predator trait $\left(\left|M_{x \beta}\right|<0\right)$ and a three-dimensional eco-coevolutionary subsystem $\left(\left|M_{x \alpha \beta}\right|>0\right)$ are unstable. Hence, that system is predicted to be unstable when predator genetic variation is sufficiently high (top left) and when genetic variation is high in both species (top right). These two examples show that increased genetic variation in one or both species can have very different effects on system stability. In addition, they also show that different subsystems may be responsible for destabilizing the system at different levels of genetic variation.

\section{Biological conditions causing subsystem instability}

The previous section showed that the effects subsystems have on the stability of the whole system depend on the prey and predator genetic variances. Importantly, if a subsystem is unstable, then that subsystem will destabilize the whole system and cause predator-prey cycles, but only when the amounts of prey and predator genetic variation are such that the subsystem has a large effect on the stability of whole system. At a phenomenological level, this is useful because it determines which components of the system are destabilizing the whole system. However, it does not identify what specific biological mechanisms cause subsystem instability. This section fills that gap by identifying the biological conditions and feedbacks that cause instability of the ecological, evolutionary, and eco-evolutionary subsystems. Details supporting the following are given in Appendix S2: Section S3. Note that smaller subsystems (with fewer variables) can destabilize larger subsystems (with more variables), e.g., the one-dimensional prey evolutionary subsystem can destabilize the two-dimensional coevolutionary subsystem. To avoid redundancy, the following only focuses on destabilizing mechanisms that do not involve instability of smaller subsystems.

Instability of the one- and two-dimensional ecological subsystems occurs via the mechanisms that drive predatorprey cycles in the absence of evolution. Mathematically, this occurs when $J_{11}>0$. One biological mechanism leading to this condition is an Allee effect in the prey at equilibrium. A second, more likely, mechanism is when the predator has a saturating functional response and overexploits its prey. In this case, the predator reduces the equilibrium prey density to the point were increased harvesting results in a decrease in predator density, e.g., past the peak of the prey nullcline in a Rosenzweig-MacArthur model (Rosenzweig and MacArthur 1963). This results in increased prey density having a positive effect on prey growth at equilibrium $\left(J_{11}>0\right)$.

The stabilities of the prey and predator evolutionary subsystems depend on whether there is stabilizing or disruptive selection in the populations. Stabilizing selection $\left(J_{33}<0\right.$, $J_{44}<0$ ) is a negative (stabilizing) evolutionary feedback, yielding stable evolutionary subsystems. In contrast, disruptive selection $\left(J_{33}>0, J_{44}>0\right)$ is a positive (destabilizing) evolutionary feedback and yields unstable evolutionary subsystems. Thus, as shown for models with a single evolving species (Cortez 2016, Cortez and Patel 2017), sufficiently large increases in genetic variation are always destabilizing under disruptive selection.

Instability of the coevolutionary subsystem occurs when $J_{34} J_{43}>0$. This condition represents a positive indirect feedback loop between the two traits $(\alpha \rightleftarrows \beta)$. There are two scenarios under which this positive feedback occurs. The more likely scenario is a coevolutionary arms race where higher offense increases the selection pressure for higher defense $\left(J_{34}>0\right)$, which in turn increases the selective pressure for higher offense $\left(J_{43}>0\right)$. This scenario represents a 

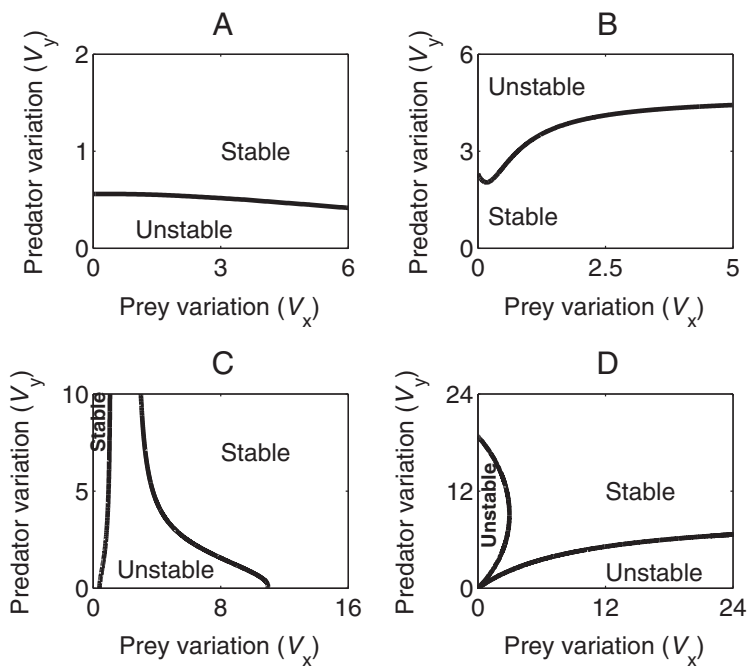

FIG. 4. Examples illustrating when different subsystems destabilize the whole system. In all panels, the black curves denote levels of genetic variation where the system changes from stable to unstable. (A) Destabilization for sufficiently low predator genetic variation due to instability of a one-dimensional ecological subsystem and a two-dimensional prey eco-evolutionary subsystem. (B) Destabilization for sufficiently high predator genetic variation due to instability of a two-dimensional predator eco-evolutionary subsystem and a three-dimensional eco-coevolutionary subsystem. (C) Destabilization for intermediate prey genetic variation when all subsystems are stable due to differences in the strengths of the stabilities of the subsystems. (D) Destabilization caused by (bottom) instability of the three-dimensional prey eco-evolutionary subsystem and (left) differences in the stabilities of stable ecological, predator evolutionary, and predator eco-evolutionary subsystems; panel recreated from Saloniemi (1993). Note that the models for (C) and (D) use different equations than model (1), but the stability theory can be applied because the models are structurally similar. See Appendix S5 for models and parameters.

coevolutionary arms race because prey defense and predator offense are both increasing. Such arms races have been observed, e.g., between phage and bacteria (Gómez et al. 2014). The less likely scenario is an "escalation-deescalation" scenario where predator offense increases while prey defense decreases, or vice versa. For example, higher offense decreases the selection pressure for higher defense $\left(J_{34}<0\right)$ and the resulting decrease in defense increases the selective pressure for higher offense $\left(J_{43}<0\right)$.

The mechanisms causing instability of the eco-evolutionary and eco-coevolutionary subsystems are similar. For ecoevolutionary subsystems involving the predator trait and eco-coevolutionary subsystems involving the predator density, instability occurs when individual predator fitness decreases with higher mean offense $\left(J_{24}<0\right)$. This can occur if high offense predators are stronger interference competitors than low offense predators; individual fitness decreases with higher mean offense because higher mean offense implies predators experience more interference competition. For example, increases in the frequency of aggressive spiders (Anelosimus studios) causes increased interference competition and reduced predator fitness through a reduction in resource-use efficiency (Pruitt and Riechert 2009). The reason this condition is destabilizing is that it results in a positive feedback between predator density and predator offense
( $y \rightleftarrows \beta$ ), defined by $J_{24} J_{42}>0$. In particular, small decreases in offense cause increases in predator fitness $\left(J_{24}<0\right)$ and the resulting increases in predator density causes further decreases in the selective pressure for offense $\left(J_{42} \leq 0\right)$. Note that this feedback is weak when intraspecific interactions between predators are weak, e.g., when predators experience scramble competition for resources and little intraspecific interference.

For eco-evolutionary subsystems involving the prey trait and eco-coevolutionary subsystems involving the prey density, instability occurs when $J_{13} J_{31}>0$. This condition is satisfied under two scenarios representing positive feedback loops between prey density and prey defense $(x \rightleftarrows \alpha)$. In scenario one, higher prey density increases the selective pressure for defense $\left(J_{31}>0\right)$, which in turn increases prey fitness $\left(J_{13}>0\right)$. The first condition $\left(J_{31}>0\right)$ is satisfied when costs for defense decrease as prey density increases. This can occur, e.g., if prey growth is modeled as $F(x, \alpha)=\left(r_{0}-\alpha\right)$ $x(1-x / K)$, where $r_{0}$ is the maximum exponential growth rate and $K$ is the prey carrying capacity; as prey density increases the costs for defense decrease to zero. The second condition $\left(J_{13}>0\right)$ is satisfied when, e.g., prey excrete chemical defenses; higher mean defense is beneficial to an individual because it means more defensive chemicals are being produced. In scenario two, increased prey density decreases the selective pressure for defense $\left(J_{31}<0\right)$ and the subsequent decrease in defense increases individual prey fitness $\left(J_{13}<0\right)$. The first condition $\left(J_{31}<0\right)$ occurs, e.g., when predators have saturating functional responses, the prey trait affects the predator-prey encounter rate, and there is frequency-dependent selection; see Eq. 2 in Frequency-dependent vs. -independent selection in the model. The second condition $\left(J_{13}<0\right)$ is satisfied when high defense prey have stronger intraspecific effects. This can occur, e.g., when prey defense is aggression and high defense prey are more aggressive towards predators and conspecifics.

Finally, instability of the eco-coevolutionary subsystems is also more likely when $J_{41}<0$, i.e., higher prey density decreases the selective pressure for offense. Here, the positive feedback driving the instability of the eco-coevolutionary subsystems is between prey density and predator offense $(x \rightleftarrows \beta$ ): increased prey density reduces the selective pressure for offense $\left(J_{41}<0\right)$ and reduced offense causes an increase in prey density $\left(J_{14}<0\right)$. The condition $J_{41}<0$ is likely to be satisfied when predators have saturating functional responses, the predator trait affects the predator-prey encounter rate, and prey density is high. For example, consider the predator numerical response $H=\alpha \beta x y /$ $(1+h \alpha \beta x)$ where the encounter rate is $\alpha \beta$ and $h$ is the handling time. As prey density increases and the predator becomes satiated, the reward for increased offense decreases, resulting in decreased selective pressure for offense.

\section{Destabilization when all subsystems are stable}

The last two sections focused on destabilization due to instability of a subsystem. This section focuses on the counter-intuitive outcome where all subsystems are stable, but the whole system is unstable. In this case, some subsystems are strongly stable while others are weakly stable, and it is the difference in the strengths of the stabilities of the 
A

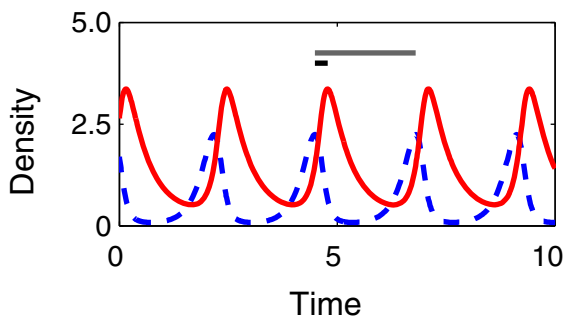

C

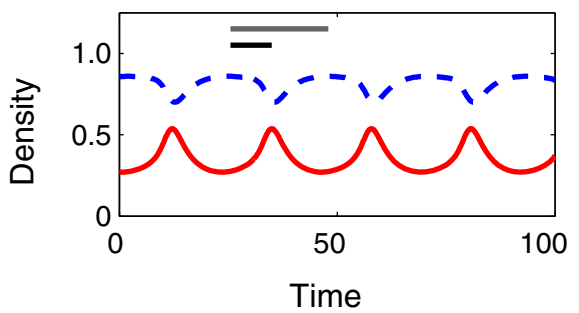

$\mathrm{E}$

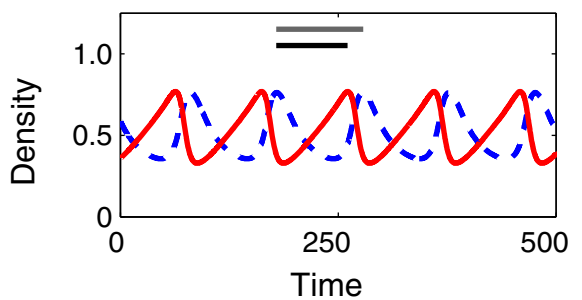

B

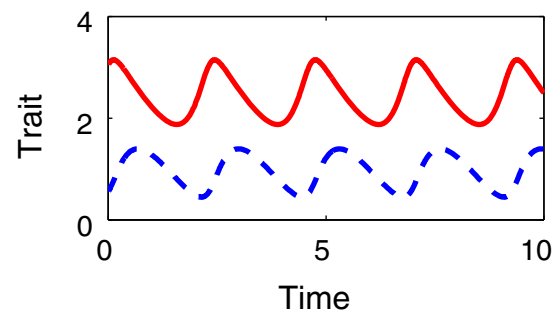

D

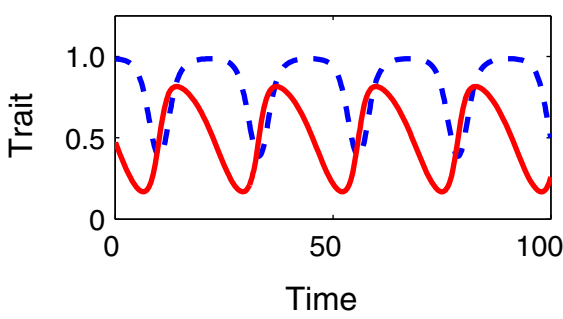

$\mathrm{F}$

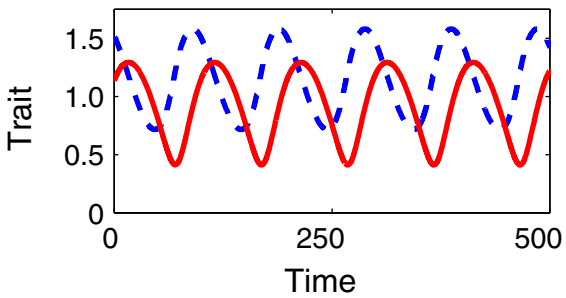

FIG. 5. Genetic variation can alter the phase lags of predator-prey cycles. Examples of cycles where the phase lags between the predator and prey oscillations are (A, B) less than one-quarter period, (C, D) between one-quarter period and one-half period, and (E, F) greater than one-half period. (A, C, E) Prey (dashed blue) and predator (solid red) densities. Gray horizontal lines show the cycle period (prey peak to prey peak) and black horizontal lines show the lag (prey peak to predator peak). (B, D, F) Mean prey defense (dashed blue) and mean predator offense (solid red). Units for densities are individual/volume and traits are unitless. See Appendix S5 for models and parameters.

subsystems that destabilizes the whole system. The conditions and mechanisms leading to destabilization are summarized below; see Appendix S2: Section S4 for details.

There are two mechanisms through which destabilization occurs due to differences in the strengths of the stabilities of the subsystems. Mechanism one corresponds to small subsystems (with fewer variables) being weakly stable compared to large subsystems (with more variables). Mathematically, this corresponds to the third term in Eq. 6 being negative or positive and small, which causes the fourth term in Eq. 6 to be negative. Mechanism two corresponds to larger subsystems being weakly stable compared to smaller subsystems. Mathematically, this corresponds to the fourth term in Eq. 6 being negative when the third term is neither small nor negative. Both mechanisms are caused by positive feedback loops. In mechanism one there is a positive feedback between the prey and predator traits ( $\alpha \rightleftarrows \beta, J_{34} J_{43}>0$; this is the coevolutionary arms race scenario from above); in mechanism two there is a positive feedback between predator density and the predator trait $\left(y \rightleftarrows \beta, J_{24} J_{42}>0\right)$; and in both mechanisms there is a positive feedback between prey density and the prey trait ( $x \rightleftarrows \alpha, J_{13} J_{31}>0$ ). These positive feedbacks are not strong enough to destabilize any of the subsystems. However, the positive feedbacks do differentially weaken the stabilities of the subsystems. This results in some subsystems being strongly stable in comparison to others, which destabilizes the whole system.

Importantly, if destabilization occurs when all subsystems are stable, then destabilization will only occur for intermediate amounts of genetic variation. For example, all subsystems are stable in Fig. 4C, yet destabilization occurs for intermediate amounts of prey genetic variation because of differences in the strengths of the stabilities of the subsystems. This means that destabilization due to an unstable subsystem and destabilization when all subsystems are stable have different signatures: unstable subsystems are destabilizing for all sufficiently large or sufficiently small amounts of genetic variation (Fig. 4A, B) whereas differences in the strengths of stability across stable subsystems are destabilizing only for intermediate levels of genetic variation in one or both species (Fig. 4C).

Finally, note that destabilization due to different strengths of stability can occur when there are unstable subsystems, provided that the instabilities of the unstable subsystems are weak. As an example, consider Fig. 4D, which was recreated from figure $2 b$ of Saloniemi (1993). For that system, only the three-dimensional prey eco-evolutionary subsystem is unstable. Thus, the whole system is unstable for sufficiently low predator genetic variation (bottom of Fig. 4D). Instability of the whole system for low prey genetic variation and 
intermediate predator genetic variation (left side of Fig. 4D) is due to mechanism 1 from above. Specifically, the ecological subsystems and two-dimensional predator eco-evolutionary subsystems are weakly stable relative to the three-dimensional predator eco-evolutionary subsystem.

\section{How genetic variation alters predator-prey phase lags}

The previous sections focused on how the magnitudes of prey and predator genetic variation influence the stability of the system. This section identifies how the amounts of prey and predator genetic variation influence the phase lags between predator and prey oscillations. I compute approximate phase lags using the Jacobian matrix, following the method in Ellner and Becks (2011); see Appendix S3 for details. This approximation is very accurate for parameter values close to Hopf bifurcations, i.e., the parameter values where the system transitions from stability to cycles (black curves in Fig. 4). However, it may not be accurate for other parameter values, particularly when cycle shape is altered by nonlocal phenomena like bistability. Thus, the following predictions provide useful approximations for the conditions that lead to different cycle types, but because they are derived from the Jacobian matrix, they do not encompass all of the biological mechanisms affecting cycle shape.

In the following, I focus on determining when three kinds of cycles occur: cycles with lags less than a quarter-period (Fig. 5A, B), cycles with lags between one-quarter and onehalf period (hereafter "antiphase cycles"; Fig. 5C, D), and cycles with lags greater than a half-period (hereafter "clockwise cycles"; Fig. 5E, F). The phase lags predicted for different amounts of prey and predator genetic variation are summarized in Table 3; letters defining the lags correspond to the panels in Fig. 5. In the following, I discuss how subsystem stability and trait-mediated indirect effects determine what kinds of cycles can arise.

The lags in predator-prey cycles are partially determined by which unstable subsystem is causing the cycles. The results in Table 3 are summarized as follows. When genetic variation is low in both species (bottom left of Fig. 3 and Table 3), cycles caused by unstable ecological subsystems have lags less than or equal to one-quarter period and cycles caused by unstable 3D eco-evolutionary subsystems can have lags of any length. When genetic variation is high in one species and low in the other (top left and bottom right of Fig. 3 and Table 3), cycles always have lags less than onehalf period. When genetic variation is high in both species (top right of Fig. 3 and Table 3), cycles caused by unstable coevolutionary subsystems can have lags of any length and cycles caused by unstable eco-coevolutionary subsystems have lags less than one-half period. Finally, when genetic variation is intermediate in at least one species, cycles caused by unstable eco-evolutionary subsystems can have lags of any length and cycles caused by unstable evolutionary subsystems have lags less than one-half period (top middle and middle right of Fig. 3 and Table 3). In total, antiphase cycles (Fig. 5B, C) can occur for any magnitudes of prey and predator genetic variation and clockwise cycles (Fig. 5B, C) can occur for any combination other than high variation in one species and low variation in the other.

Trait-mediated indirect effects of the prey and predator densities on the predator dynamics determine why phase lags differ depending on which unstable subsystem is causing the cycles. In predator-prey models without evolution, lags less than one-quarter period are caused by the direct effects defined by $J_{21}>0$ and $J_{21} J_{22}<0$ (Bulmer 1975). Here, $J_{21}$ represents the positive direct effect of increased prey density on the predator dynamics $(x \rightarrow y)$ and $J_{21} J_{22}$ represents the product of that direct effect and the negative direct self-effect of the predator on its own dynamics $(x \rightarrow y \circlearrowleft)$. For the eco-coevolutionary model (1), the conditions determining predator-prey phase lags are more complex and involve many more terms; see equations S7-S10 in Appendix S3: Section S2. However, all of the additional terms represent trait-mediated indirect effects of the prey and predator densities on the predator dynamics. For example, $J_{23} J_{31}$ represents the indirect effect of prey density on the predator dynamics mediated by the prey trait $(x \rightarrow \alpha \rightarrow y)$ and $J_{23} J_{32} J_{21}$ includes an indirect self-effect of the predator mediated by the prey trait $(x \rightarrow y \rightleftarrows \alpha)$. Traitmediated indirect effects can cause an increase or decrease in the predator-prey phase lag. Trait-mediated indirect effects of prey density on the predator dynamics (e.g., $x \rightarrow \alpha \rightarrow y$ ) promote lags greater than one-quarter period when they are positive and larger than the direct effect. This is because the indirect effects introduce a lagged response:

TABLE 3. Phase lags predicted for different magnitudes of prey and predator genetic variation.

\begin{tabular}{|c|c|c|c|c|c|c|}
\hline \multirow[b]{3}{*}{ Predator (pred) genetic variation } & \multicolumn{6}{|c|}{ Prey genetic variation } \\
\hline & \multicolumn{2}{|l|}{ Low } & \multicolumn{2}{|l|}{ Intermediate } & \multicolumn{2}{|l|}{ High } \\
\hline & Unstable subsystem & Lag & Unstable subsystem & Lag & Unstable subsystem & Lag: \\
\hline \multirow[t]{2}{*}{ High } & Pred Evo & $\mathrm{AC}$ & Pred Evo & $\mathrm{AC}$ & Coevo & $\mathrm{ACE}$ \\
\hline & 2D, 3D Pred Eco-Evo & $\mathrm{AC}$ & $\begin{array}{l}\text { 2D, 3D Pred Eco-Evo, } \\
\text { Eco-Coevo, Coevo }\end{array}$ & ACE & Eco-Coevo & $\mathrm{AC}$ \\
\hline \multirow[t]{2}{*}{ Intermediate } & 2D, 3D Pred Eco-Evo & $\mathrm{ACE}$ & Any or none $\dagger$ & ACE & Prey Evo & $\mathrm{AC}$ \\
\hline & & & & & $\begin{array}{l}\text { 2D, 3D Prey Eco-Evo, } \\
\text { Eco-Coevo, Coevo }\end{array}$ & $\mathrm{ACE}$ \\
\hline \multirow[t]{2}{*}{ Low } & 2D Ecological & A & 2D, 3D Prey Eco-Evo & ACE & Prey Evo & $\mathrm{AC}$ \\
\hline & 3D Eco-evo & ACE & & & 2D, 3D Prey Eco-Evo & A \\
\hline
\end{tabular}

Note: Evo, evolutionary; Eco-Evo, eco-evolutionary; Coevo, coevolutionary; Eco-Coevo, eco-coevolutionary.

$\dagger$ For intermediate variation in both species, cycles can be driven by any unstable subsystem or differences in the stabilities of all stable subsystems.

\$Letters for phase lags reference examples in Fig. 5: (A) lags less than one-quarter period, (C) lags between one-quarter and one-half period, and (E) lags greater than one-half period. 
increased prey density first causes an evolutionary response in one or both species, and then that evolutionary responses causes an increase in predator density. When the lagged response to the indirect effect is larger than the response to the direct effect, the timing of the predator peak is delayed, causing an increase in the lag. Trait-mediated indirect predator self-effects (e.g., $x \rightarrow y \rightleftarrows \alpha$ ) promote lags greater than one-quarter period when they are positive and larger than the direct effect. This is because the indirect effects decrease the negative self-effects of the predator, which allows the predator population to increase for a longer period of time, delaying the predator peak.

As illustrative examples, below I present a few traitmediated indirect effects that have a large influence when prey genetic variation is greater than or equal to predator genetic variation (i.e., regions of Fig. 4 on or below the oneto-one line). A full list of all of the indirect effects and their regions of influence in parameter space are given in Appendix S3: Section S2. Note that when genetic variation is intermediate in both species, all of the indirect effects listed below and in Appendix S3: Section S2 influence predator-prey phase lags.

First consider systems where prey and predator genetic variation are both low (bottom left of Fig. 4). Cycles caused by unstable ecological subsystems have lags less than onequarter period (Fig. 5A, B) because the predator oscillations are primarily driven by the direct effects $J_{21}(x \rightarrow y)$ and $J_{21} J_{22}(x \rightarrow y \circlearrowleft)$. In contrast, cycles caused by three-dimensional eco-evolutionary subsystems can have lags of any length because the predator oscillations are altered by many different trait-mediated indirect effects; see Appendix S3: Section S2 for details.

Now consider systems where prey genetic variation is high and predator genetic variation is low (bottom right of Fig. 3). When the cycles are caused by an unstable prey evolutionary subsystem, antiphase cycles are driven the indirect effect of prey density on the predatory dynamics mediated by the prey trait $(x \rightarrow \alpha \rightarrow y)$. The mathematical condition is $J_{23} J_{31}>0$. Biologically, this occurs when increases in prey density decrease the selective pressure for defense $\left(J_{31}<0\right)$, e.g., low defense prey are stronger intraspecific competitors. These conditions imply that increases in prey density are followed by a decrease in mean defense $\left(J_{31}<0\right)$, which is then followed by an increase in predator density $\left(J_{23}<0\right)$. Thus, in these cycles, peaks in mean defense are followed by peaks in prey density, which are followed by peaks in predator density (Fig. 5C, D). In contrast, when the prey evolutionary subsystem is stable (implying stabilizing selection) and cycles are caused by unstable prey eco-evolutionary subsystems, the cycle lag is determined by the trait-mediated indirect effects in the $y, \alpha$ subsystem. Under stabilizing selection, those trait-mediated indirect effects always cause the lags to be less than one-quarter period.

Next, consider systems where prey genetic variation is intermediate and predator genetic variation is low (bottom middle of Fig. 4). In these systems, cycles must be caused by unstable prey eco-evolutionary subsystems. Antiphase cycles are caused by the condition in the previous paragraph. Clockwise cycles arise via the conditions $J_{22} J_{23} J_{31}>0$ and $-J_{23} J_{32} J_{21}>0$. Those conditions describe how prey traitmediated indirect effects alter the effect of prey density on the predator dynamics $(x \rightarrow \alpha \rightarrow y \circlearrowleft)$ and the self-effect of the predator $(x \rightarrow y \rightleftarrows \alpha)$, respectively. The first condition reduces to $J_{31}>0$, which is the opposite of the condition for antiphase cycles. The second condition is always positive, implying that clockwise cycles can arise if there is a strong feedback loop between predator density and prey defense.

Finally, consider systems where genetic variation is high in both species (top right of Fig. 5G). When the cycles are caused by an unstable coevolutionary subsystem, clockwise cycles are driven by the indirect effects of prey density on the predator dynamics mediated by both traits $(x \rightarrow \alpha \rightarrow \beta \rightarrow y$ and $x \rightarrow \beta \rightarrow \alpha \rightarrow y$ ); the mathematical conditions are $J_{24} J_{43} J_{31}>0$ and $J_{23} J_{34} J_{41}>0$. Both of these indirect effects are three-step processes that introduce a delayed positive response to increased prey density and cause the lag to be greater than one-half period. In the high genetic variation limit, these cycles are more likely to arise when increased offense decreases the selection pressure for defense $\left(J_{34}<0\right)$ and increased defense increases the selection pressure for offense $\left(J_{43}>0\right)$; these conditions correspond to the "prey escalates first" scenario in Cortez (2015), where increased defense is followed by increased offense. Altogether, the conditions predict that in clockwise cycles, the order of the peaks is prey density, mean prey defense, mean predator offense, and finally predator density (Fig. 5E, F). In contrast, when the coevolutionary subsystem is stable and the cycles are caused by unstable eco-coevolutionary subsystems, all trait-mediated indirect effects cause the lag to be less than a half-period. Thus, when genetic variation is high in both species, unstable coevolutionary dynamics are necessary for clockwise cycles.

\section{Discussion}

In this study, I explored how standing genetic variation influences the stability and cyclic dynamics of predator-prey systems. My results show how the magnitudes of prey and predator genetic variation dictate which components (i.e., subsystems or feedback loops) of the system influence the stability of the whole system (Fig. 3). My results also show how the magnitudes of genetic variation influence the phase lags in predator-prey cycles by altering the strengths of trait-mediated indirect effects of prey and predator density on the predator population dynamics. These results help identify why altered genetic variation caused stability changes in empirical systems and identify the mechanisms driving cyclic dynamics in those systems. They also unify and extend the existing body of theory on the eco-evolutionary dynamics of predator-prey systems.

\section{Effects of altered genetic variation on system stability}

This theory helps explain why increased genetic variation in one species altered the dynamics of two predator-prey systems. In a rotifer-algae system (Becks et al. 2010), increased prey genetic variation was destabilizing and caused antiphase cycles. In that system, predator genetic variation was low. Hence, only the subsystems in the bottom half of Fig. 3 influenced the stability of the system. Because the system is stable for low prey genetic variation, the ecological subsystems and the three-dimensional eco-evolutionary subsystems 
involving a single trait must have been stable. In contrast, because cyclic dynamics occurred for high prey genetic variation, the instability of the whole system must have been due to instability of either the prey evolutionary subsystem or the two-dimensional eco-evolutionary subsystem involving the prey trait. Previous theoretical work on this system predicts disruptive selection in the algal population (Jones and Ellner 2004, 2007, Becks et al. 2010), implying that the prey evolutionary subsystem was unstable $\left(J_{33}>0\right)$. This means that the eco-evolutionary cycles in that system were driven, at least in part, by a positive prey evolutionary feedback. In also suggests that the eco-evolutionary cycles could have been driven solely by a positive evolutionary feedback and not an eco-evolutionary feedback.

In a ciliate-bacteria system (Hiltunen and Becks 2014), the population dynamics differed depending on the selection history of the predator. When predators who had not been exposed to defended bacterial prey (un-evolved predators) were used, the system converged to a steady state. In contrast, when evolved or co-evolved predators who had been previously exposed to defended bacteria were used, the system did not converge to a steady state. Bacterial standing genetic variation was expected to be high in all treatments. In addition, the evolutionary histories of the predator populations suggest that the magnitudes of standing genetic variation were low in the un-evolved predator populations and higher in the evolved and co-evolved predator populations (Hiltunen and Becks 2014). Taken together, this suggests that only the subsystems in the right half of Fig. 3 influenced the stability of the system. Because the system was stable when predator genetic variation was low, the prey evolutionary and eco-evolutionary subsystems must have been stable. Thus, my theory predicts that destabilization of the communities with evolved and co-evolved predator populations was due to instability of the predator evolutionary, coevolutionary, and/or eco-coevolutionary subsystems. The data and experiments in Hiltunen and Becks (2014) do not allow one to rule out any of the three possible subsystems. However, my theory identifies specific experiments that can be used to distinguish between the three possibilities. For example the stabilities of the predator evolutionary and coevolutionary subsystems can be determined via selection experiments targeting stabilizing vs. disruptive selection in the ciliate population (to determine the sign of $J_{44}$ ) and the evolutionary responses of both species to increased offense/defense in the other population (to determine the signs of $J_{34}$ and $J_{43}$ ).

It is important to note that the above predictions implicitly assume that the mean prey and predator trait values are the same for low and high genetic variation populations. Since the mean trait values are likely to differ to some degree between low and high genetic variation populations, increased genetic variation in one or both populations may not be the sole reason destabilization occurs in the two empirical systems. For example, destabilization in the ciliatebacteria system could also have been due to the evolved and co-evolved predators having evolved higher attack rates on defended bacteria. Such a change in attack rate would alter the stabilities of one or more subsystems, e.g., increase the instability of the three-dimensional predator eco-evolutionary subsystem. Thus, increased predator genetic variation could have had destabilizing effects through both changes in the relative strengths of all subsystems (as predicted by the theory in this study) and changes in the stabilities of specific subsystems (due to changes in the mean predator trait).

The stability theory developed in this paper is mathematically identical to Levins' loop analysis (Levins 1974). The above empirical examples illustrate one advantage of interpreting the stability conditions in terms of both subsystems and feedback loops. A natural experimental approach is to hold one variable or factor (nearly) constant and observe the dynamics of the remaining variables. The dynamics of this partially controlled system correspond to the dynamics of a subsystem, e.g., the ciliate-bacteria chemostat with unevolved predators corresponds to the three-dimensional prey eco-evolutionary subsystem. In contrast, it is more difficult to manipulate a feedback loop without altering other aspects of the system. A second advantage is that the mathematical conditions and terms in the Routh-Hurwitz criteria (Eqs. 6 and 7) are more naturally analyzed and interpreted in terms of subsystem stability. Thus, the subsystem perspective facilitates connections between the biology, experiments, and theory. That being said, it is important to note that feedback loops are the underlying determinants of subsystem stability. For example, the stability of the coevolutionary subsystem is determined by the feedback loops involving one $\left(J_{33}, J_{44}\right)$ or both $\left(J_{34} J_{43}\right)$ traits. Thus, while subsystems are a natural way to interpret the theory, the underlying mechanisms are defined by feedback loops.

Regardless of which interpretation is used, the stability theory developed in this paper explains why previous theoretical studies on coevolutionary predator-prey models have found differing effects of increased genetic variation in one or both species. (Calculations and additional details supporting the following are given in Appendix S4: Section S2). Some previous studies have reported destabilization with increased prey genetic variation. Across those studies, destabilization was due to either instability of the prey evolutionary subsystem (i.e., disruptive selection; Abrams and Matsuda 1997a, Mougi and Iwasa 2011, Mougi 2012a), instability of a prey eco-evolutionary subsystem (Saloniemi 1993), or both (Mougi and Iwasa 2011). In contrast, Tien and Ellner (2012) observed destabilization for intermediate levels of prey genetic variation (Fig. 5 of that study). The result in Tien and Ellner (2012) was driven by a different mechanism: all subsystems were stable and instability for intermediate genetic variation was due to differences in the strengths of the stabilities of the ecological, prey evolutionary, and prey eco-evolutionary subsystems.

Previous studies have also reported differing results for the effects of increased predator genetic variation on stability in coevolutionary predator-prey models; stabilization is common (Saloniemi 1993, Abrams and Matsuda 1997a, Mougi and Iwasa 2011, Mougi 2012a, Tien and Ellner 2012), but destabilization has also been observed (Mougi and Iwasa 2011). My results reveal the underlying causes for the different predictions. Specifically, in all cases where stabilization was observed, the ecological subsystems were strongly stable and all subsystems involving the predator trait were stable. Hence, the systems were stabilized with increased predator genetic variation. In contrast, the predator eco-evolutionary subsystem was unstable or weakly stable in Mougi and Iwasa (2011) due to (1) a positive 
feedback between the prey density and the predator trait $\left(J_{14} J_{41}>0\right)$ and (2) instability or weak stability of the ecological subsystem. Hence, in that model, increased predator genetic variation was destabilizing.

Importantly, in all of the above studies the effects of increased prey or predator genetic variation were nonlinear, i.e., the effect of increased genetic variation in one species depended on the amount of genetic variation in the other species. For example, in Fig. 4D (recreated from Saloniemi 1993) increased prey genetic variation is destabilizing when predator genetic variation is low (bottom half of Fig. 4D), stabilizing when predator genetic variation is intermediate (middle of Fig. 4D), and does not affect stability when predator genetic variation is high (top half of Fig. 4D). Fig. 3 shows that this context dependence should not be surprising: varying the amounts of genetic variation in one or both species changes which subsystems influence the stability of the whole system.

This collection of studies highlights how the subsystem stability theory in this study can be used to identify what underlying mechanisms in models are driving seemingly contradictory predictions about the effects of altered genetic variation. This in turn can help explain the patterns and dynamics in empirical systems. For example, applying this theory to parameterized models of empirical systems could help explain why population cycles were observed in some coevolutionary predator-prey systems (Mizoguchi et al. 2003, Haafke et al. 2016) but not others (Frickel et al. 2016). It may also help explain why changes in environmental conditions (e.g., chemostat dilution rates) caused changes in system stability (Mizoguchi et al. 2003).

\section{Effects of altered genetic variation on eco-evolutionary cycles}

Ecological models without evolution predict that peaks in predator abundance lag behind peaks in prey abundance by one-quarter period or less (Bulmer 1975). In contrast, empirical systems and predator-prey models with prey evolution exhibit cycles with lags up to one-half period, including antiphase cycles (one-half period lags; Yoshida et al. 2003, Cortez 2016) and cryptic cycles (one species oscillates while the other is effectively constant; Jones and Ellner 2007, Yoshida et al. 2007). Predator evolution is also predicted to drive such cycles, but it is less likely to do so since those cycles require the system to satisfy more restrictive biological conditions (Cortez and Ellner 2010, Cortez and Patel 2017). Finally, coevolution is predicted to cause cycles with lags up to a half-period (Mougi and Iwasa 2010, 2011, Cortez 2015) as well as cycles with lags greater than one-half period called clockwise cycles (Cortez and Weitz 2014). This body of work suggests that predator-prey phase lags can be used as signatures of evolution in one or both species. Indeed, previous studies have identified empirical systems exhibiting antiphase cycles (Hiltunen et al. 2014) or clockwise cycles (Cortez and Weitz 2014) and argued that prey evolution or coevolution are likely mechanisms driving those dynamics.

The phase lag theory in this study adds to this body of work in two key ways. First, the above theoretical studies have identified many specific biological conditions under which evolution and coevolution alter predator-prey cycles. This study shows that the underlying mechanisms that lead to altered phase lags are trait-mediated indirect effects of prey and predator density on the predator population dynamics. Second, the theory presented in Appendix S3 shows that there are many different trait-mediated indirect effects that can alter predator-prey phase lags. Consequently, the specific mechanisms causing altered lags are likely to differ between systems. Nonetheless, the theory in this study helps identify when those mechanisms are likely to be important. This in turn identifies the limitations of predicted mechanisms of antiphase and clockwise cycles from previous studies.

Table 3 shows that antiphase cycles can occur for any magnitudes of prey and predator genetic variation. Previous studies (Jones and Ellner 2007, Yoshida et al. 2007, Cortez and Ellner 2010, Cortez 2016) predict that antiphase cycles are likely when defense is costly in terms of large reductions in intraspecific competitive ability ( $J_{31}$ negative and large in magnitude) and the prey experience disruptive selection $\left(J_{33}>0\right)$. This study shows that the underlying mechanism for that prediction is an indirect effect of prey density on the predator dynamics mediated by the prey trait $(x \rightarrow \alpha \rightarrow y$, $\left.J_{23} J_{31}>0\right)$. The results in this study also show that this trait-mediated indirect effect can cause antiphase cycles only when prey genetic variation is (1) intermediate or high and (2) comparable to or greater than predator genetic variation (i.e., on or below the one-to-one line in Fig. 3). For low genetic variation in both species (bottom left of Fig. 3), Mougi and Iwasa (2011) proposed that antiphase cycles must be driven by evolution when the system is stable in the absence of evolution (i.e., the ecological subsystems are stable). In addition to identifying the specific trait-mediated indirect effects that cause antiphase cycles when genetic variation is low in both species, this study provides additional support for that prediction. In particular, antiphase cycles are not predicted when genetic variation is low in both species if the ecological subsystem is unstable (only lags less than a quarter-period are predicted; bottom left of Table 3). Instead, antiphase cycles are only possible when the ecological subsystem is stable and an unstable three-dimensional eco-evolutionary subsystem is causing the cycles.

Previous studies by the author (Cortez and Weitz 2014, Cortez 2015) predict clockwise cycles occur under particular biological conditions. This study shows that those conditions are not necessary for clockwise cycles. For example, sufficiently high genetic variation in both species was predicted to be a necessary condition for clockwise cycles. This study predicts that clockwise cycles can occur for any amounts of genetic variation other than combinations where one species has very high genetic variation and the other has very low genetic variation (top left and bottom right corners of Table 3). Note that this prediction is supported by a recent numerical study (van Velzen and Gaedke 2017) where unrecognized examples of clockwise cycles occurred when genetic variation was low in both species (see next paragraph). Disruptive selection in both species was also predicted to be a necessary condition for clockwise cycles. This study shows that is not the case. First, in the fast evolution limit (top right of Table 3), the true necessary condition for clockwise cycles is an unstable coevolutionary subsystem. This can occur via disruptive selection in one or both species. Second, disruptive selection is not a necessary 
condition for clockwise cycles when genetic variation is intermediate or low in one or both species (see conditions in Appendix S3). In combination, the above highlights how the phase lag theory in this study can be used to extend and give additional context to results from previous studies.

The main advantage of the phase lag theory in this study is that it allows one to make analytical predictions about the mechanisms driving different types of eco-evolutionary cycles. However, it is important to keep in mind that the predictions from the method are limited because they are only guaranteed to be accurate for parameter values close to the transitions from stability to cycles (known as Hopf bifurcations; black curves in Fig. 4). For example, van Velzen and Gaedke (2017) used numerical simulations to explore how the magnitudes of prey and predator genetic variation influenced the occurrence of antiphase cycles. When applied to their model (see Appendix S3: Section S3 for details), the phase lag theory in this study accurately predicted a transition from antiphase cycles to cycles with one-quarter period lag as predator genetic variation increased. The theory also accurately predicted that all antiphase cycles that arise for sufficiently low prey and predator genetic variation have a clockwise orientation (the reversed cycle orientation was not recognized in the original study). The accuracy of these predictions is not surprising because both predictions were made for parameter values close to Hopf bifurcations. The theory in this study also predicted, for parameter values far from Hopf bifurcations, that increased prey genetic variation would cause a transition from antiphase cycles to quarter-period lag cycles. In contrast, in simulations, increased prey genetic variation only caused a small decrease in the phase lag (van Velzen and Gaedke 2017). Thus, while the theory correctly predicted the trend (decreased lag with increased prey genetic variation), it greatly overestimated the size of the effect. In total, the theory is a useful starting point for studying eco-evolutionary cycles, but because it is based on an approximation, it is unlikely to explain all patterns observed across systems.

This study and the body of work cited above focus on interpreting antiphase and clockwise cycles in terms of signatures of (co)evolution in predator-prey systems. However, it is important to note that alternative mechanisms could be driving those cycles (Barraquand et al. 2017). Previous studies (e.g., Abrams 2006, Mougi 2012b) have modeled induced plastic change using models virtually identical to model (1). While plasticity in one species is not predicted to drive cycles with a lag greater than one-quarter period (Cortez 2011), those studies suggest that co-plasticity could potentially drive cycles with longer lags (Mougi 2012b). Stage structure in the prey or the predator can also alter phase lags (e.g., De Roos et al. 1990, De Roos and Persson 2003). Thus, while predator-prey phase lags may indicate evolution as a driving mechanism, it is important to use caution and rule out other possible driving mechanisms.

\section{Connections with other bodies of theory}

The theory developed in this study helps build connections between existing bodies of theory on eco-evolutionary dynamics. Previous studies have developed theory identifying when and whether eco-evolutionary feedbacks stabilize or destabilize predator-prey systems. To simplify the mathematical analysis, those studies have focused on the cases where only only species was evolving (Cortez 2016, Cortez and Patel 2017), the ecological dynamics were much faster than the evolutionary dynamics (which includes the theory of adaptive dynamics; Dieckmann et al. 1995, Marrow et al. 1996, Geritz et al. 1998), or the evolutionary dynamics were much faster than the ecological dynamics (Cortez and Ellner 2010; Patel et al., 2018). The results in those studies can be unified using the subsystem stability theory in this study.

Two recent studies on models with a single evolving species explored how increased prey (Cortez 2016) or predator (Cortez and Patel 2017) genetic variation altered the stability of predator-prey systems. Those studies correspond to the horizontal and vertical axes in Fig. 3 where one species has no genetic variation $\left(V_{x}=0\right.$ or $\left.V_{y}=0\right)$. The mathematical conditions determining system stability and predator-prey phase lags for systems with low genetic variation in one species (e.g., $V_{y} \approx 0$ ) versus no genetic variation (e.g., $V_{y}=0$ ) are largely the same. However, there are two important differences that arise. First, models with a single evolving species predict that clockwise cycles are virtually impossible because the mathematical conditions for those cycles are very restrictive (Cortez 2016, Cortez and Patel 2017). In contrast, clockwise cycles are possible in coevolutionary models where genetic variation is low in one species and intermediate in the other (Table 3). Second, models with a single evolving species predict antiphase cycles can only occur when genetic variation is sufficiently high in the evolving species. However, the results in Mougi and Iwasa (2011) and this study show that antiphase cycles can arise when genetic variation is low in both species. This disagreement between evolutionary and coevolutionary models is caused by the loss/gain of subsystems. For example, when genetic variation is low in both species in a coevolutionary model, stability of the whole system is determined by the ecological and three-dimensional eco-evolutionary subsystems. In contrast, when genetic variation is low in a model with one evolving species, stability of the whole system is determined solely by the ecological subsystems. Thus, low genetic variation $\left(V_{y} \approx 0\right)$ and no genetic variation $\left(V_{y}=0\right)$ are different because the latter removes all feedbacks involving the predator trait. This helps identify the limitations of the predictions from models with a single evolving species and how to unify them with results from coevolutionary systems.

Subsystem stability theory also helps unify results from theory with an assumed separation time scales between ecological and evolutionary processes. Consider the slow evolution limit where ecological dynamics are much faster than evolutionary dynamics. This limit corresponds to the bottom left corner of Fig. 3 where genetic variation is low in both species. The ecological feedbacks determine the stability of the fast ecological dynamics of the system. Therefore, the stability of the (slower) eco-evolutionary dynamics of the system must be determined by the stability of the ecoevolutionary subsystems. Now consider studies on the fast evolution limit, where evolutionary dynamics are faster than ecological dynamics. The fast evolution limit corresponds to the top right corner of Fig. 3 where genetic variation is high in both species. In this limit, evolutionary and coevolutionary subsystems determine the stability of the fast evolutionary dynamics and the eco-coevolutionary subsystems 
determine the stability of the (slower) eco-evolutionary dynamics of the system.

What this means is that these two limits are providing information about different eco-evolutionary feedbacks. Specifically, studies on the slow evolution limit provide information about the dynamics driven by three-dimensional eco-evolutionary feedbacks that involve a single trait. In contrast, studies on the fast evolution limit provide information about the dynamics driven by eco-coevolutionary feedbacks involving both traits. This is important for two reasons. First, it shows that the fast and slow evolution approaches complement each other by providing information about different ways in which eco-evolutionary feedbacks alter population-level ecological and evolutionary dynamics. Thus, it is useful to consider both limits when trying to understand eco-evolutionary dynamics. Second, it identifies the limitations of those approaches. Specifically, the fast and slow evolution limits do not provide insight about the two-dimensional eco-evolutionary feedbacks involving a single trait (terms in $a_{2}$ of Eq. 7). The fast and slow evolution limits also do not provide information about what stability changes can occur for intermediate amounts of genetic variation, e.g., the fast and slow evolution limits cannot explain or even determine that stability changes occur in Fig. 4C. Thus, while the analytical tractability of the fast and slow evolution limits makes them useful starting points, they may not provide a complete picture.

Finally, while this study has focused on the eco-evolutionary dynamics of predator-prey systems, adapting this theory to other contexts will likely be fruitful. One obvious area is eco-evolutionary dynamics in other interaction webs. For example, altered genetic variation has been observed to influence species coexistence and stability in empirical (Lankau and Strauss 2007, Agashe 2009, Clark 2010) and theoretical (Fox and Vasseur 2008, Vasseur et al. 2011, Mougi 2013) competitive systems. A second area where subsystem stability theory could also be useful is in clarifying when and if phenotypic plasticity and evolution have different effects on population-level dynamics and system stability. Previous theoretical work on rapid plastic responses predicts that plasticity is stabilizing and decreases predator-prey phase lags (Cortez 2011). However, those predictions may not hold if induction is delayed (Underwood 1999) or if the induced phenotype persists after removal of the stimulus (Kopp and Gabriel 2006). Predictions may also differ depending on whether the plastic response is assumed to follow the fitness gradient (Kondoh 2003, Abrams 2006, Mougi 2012b) or not (Vos et al. 2004a, b, Cortez 2011). A third area is the dynamics of systems with multiple species at each trophic level. Because model (1) is a useful approximation for studying clonal species with discrete trait values (Abrams and Matsuda 1997b, Cortez and Weitz 2014), similar approaches may be useful for studying trophic-level cycles (e.g., cycles in total prey and total predator abundances). In this framework, different cycle types could suggest fluctuations in the relative abundances of species within a trophic level.

\section{ACKNOWLEDGMENTS}

I thank P. Abrams, the Duffy Lab at the University of Michigan, and two reviewers for very helpful and insightful comments on previous versions of the manuscript.

\section{Literature Cited}

Abrams, P. A. 1986. Character displacement and niche shift analyzed using consumer-resource models of competition. Theoretical Population Biology 29:107-160.

Abrams, P. A. 1992. Adaptive foraging by predators as a cause of predator-prey cycles. Evolutionary Ecology 6:56-72.

Abrams, P. A. 2000. The evolution of predator-prey interactions: Theory and evidence. Annual Review of Ecology, Evolution and Systematics 31:79-105.

Abrams, P. A. 2006. The prerequisites for and likelihood of generalist-specialist coexistence. American Naturalist 167:329-342.

Abrams, P. A., and H. Matsuda. 1997a. Fitness minimization and dynamic instability as a consequence of predator-prey coevolution. Evolutionary Ecology 11:1-20.

Abrams, P. A., and H. Matsuda. 1997b. Prey adaptation as a cause of predator-prey cycles. Evolution 51:1742-1750.

Abrams, P. A., H. Matsuda, and Y. Harada. 1993. Evolutionarily unstable fitness maxima and stable fitness minima of continuous traits. Evolutionary Ecology 7:465-487.

Agashe, D. 2009. The stabilizing effect of intraspecific genetic variation on population dynamics in novel and ancestral habitats. American Naturalist 174:255-267.

Barraquand, F., et al. 2017. Moving forward in circles: challenges and opportunities in modelling population cycles. Ecology Letters 20:1074-1092.

Becks, L., S. P. Ellner, L. E. Jones, and N. G. H. Jr. 2010. Reduction of adaptive genetic diversity radically alters eco-evolutionary community dynamics. Ecology Letters 13:989-997.

Bolnick, D. I., P. Amarasekare, M. S. Araújo, R. Bürger, J. M. Levine, M. Novak, V. H. W. Rudolf, S. J. Schreiber, M. C. Urban, and D. A. Vasseur. 2011. Why intraspecific trait variation matters in community ecology. Trends in Ecology and Evolution 26:183192.

Brodie III, E. D., and E. D. Brodie Jr. 1999. Costs of exploiting poisonous prey: Evolutionary trade-offs in a predator-prey arms race. Evolution 53:626-631.

Bulmer, M. G. 1975. Phase relations in the ten-year cycle. Journal of Animal Ecology 44:609-621.

Cannon, R. E., M. S. Shane, and J. M. Whitaker. 1976. Interaction of Plectonema boryanum (cyanophycae) and LPP-cyanophages in continuous culture. Journal of Phycology 12:418-421.

Clark, J. S. 2010. Individuals and the variation needed for high species diversity in forest trees. Science 327:1129-1132.

Coberly, L. C., W. Wei, K. Y. Sampson, J. Millstein, H. A. Wichman, and S. M. Krone. 2009. Space, time, and host evolution facilitate coexistence of competing bacteriophages: Theory and experiment. American Naturalist 173:E121-E138.

Cortez, M. H. 2011. Comparing the qualitatively different effects rapidly evolving and rapidly induced defences have on predatorprey interactions. Ecology Letters 14:202-209.

Cortez, M. H. 2015. Coevolution-driven predator-prey cycles: predicting the characteristics of eco-coevolutionary cycles using fast-slow dynamical systems theory. Theoretical Ecology 8:369382.

Cortez, M. H. 2016. How the magnitude of prey genetic variation alters predator-prey eco-evolutionary dynamics. American Naturalist 188:329-341.

Cortez, M. H., and P. A. Abrams. 2016. Hydra effects in stable communities and their implications for system dynamics. Ecology 97:1135-1145.

Cortez, M. H., and S. P. Ellner. 2010. Understanding rapid evolution in predator-prey interactions using the theory of fast-slow dynamical systems. American Naturalist 176:E109-E127.

Cortez, M. H., and S. Patel. 2017. The effects of predator evolution and genetic variation on predator-prey population-level dynamics. Bulletin of Mathematical Biology 79:1510-1538.

Cortez, M. H., and J. S. Weitz. 2014. Coevolution can reverse predator-prey cycles. Proceedings of the National Academy of Sciences USA 111:7486-7491. 
Crutsinger, G. M., M. D. Collins, J. A. Fordyce, Z. Gompert, C. C. Nice, and N. J. Sanders. 2006. Plant genotypic diversity predicts community structure and governs an ecosystem process. Science 313:966-968.

De Roos, A. M., and L. Persson. 2003. Competition in sizestructured populations: mechanisms inducing cohort formation and population cycles. Theoretical Population Biology 63:116.

De Roos, A. M., J. A. J. Metz, E. Evers, and A. Leipoldt. 1990. A size dependent predator-prey interaction: who pursues whom? Journal of Mathematical Biology 28:609-643.

Dieckmann, U., P. Marrow, and R. Law. 1995. Evolutionary cycling in predator-prey interactions: population dynamics and the Red Queen. Journal of Theoretical Biology 176:91-102.

Doebeli, M. 1997. Genetic variation and the persistence of predator-prey interactions in the Nicholson-Bailey model. Journal of Theoretical Biology 188:109-120.

Edelstein-Keshet, L. 1989. Mathematical models in biology. McGraw-Hill, New York, New York, USA.

Ellner, S. P., and L. Becks. 2011. Rapid prey evolution and the dynamics of two-predator food webs. Theoretical Ecology 4:133152.

Fox, J. W., and D. A. Vasseur. 2008. Character convergence under competition for nutritionally essential resources. American Naturalist 172:667-680.

Frickel, J., M. Sieber, and L. Becks. 2016. Eco-evolutionary dynamics in a coevolving host-virus system. Ecology Letters 19:450-459.

Fridley, J. D., and J. P. Grime. 2010. Community and ecosystem effects of intraspecific genetic diversity in grassland microcosms of varying species diversity. Ecology 91:2272-2283.

Gantmacher, F. R. 1998. The theory of matrices. Volume 131. American Mathematical Society Chelsea Publishing Company, Providence, Rhode Island, USA

Gause, G. F. 1935. Experimental demonstration of Volterra's periodic oscillations in the numbers of animals. Journal of Experimental Biology 12:44-48.

Geritz, S. A. H., É. Kisdi, G. Meszéna, and J. A. J. Metz. 1998. Evolutionarily singular strategies and the adaptive growth and branching of the evolutionary tree. Evolutionary Ecology 12:3557.

Gómez, P., B. Ashby, and A. Buckling. 2014. Population mixing promotes host-parasite coevolution. Proceedings of the Royal Society B 282:20142297.

Haafke, J., M. A. Chakra, and L. Becks. 2016. Eco-evolutionary feedback promotes Red Queen dynamics and selects for sex in predator populations. Evolution 70:641-652.

Hiltunen, T., and L. Becks. 2014. Consumer co-evolution as an important component of the eco-evolutionary feedback. Nature Communications 5:5226.

Hiltunen, T., S. P. Ellner, G. Hooker, L. E. Jones, and N. G. Hairston Jr. 2014. Eco-evolutionary dynamics in a three-species food web with intraguild predation: Intriguingly complex. Advances in Ecological Research 150:41-74.

Imura, D., Y. Toquenaga, and K. Fujii. 2003. Genetic variation can promote system persistence in an experimental host-parasitoid system. Population Ecology 45:205-212.

Ingram, T., W. E. Stutz, and D. I. Bolnick. 2011. Does intraspecific variation in a predator affect diet diversity and top-down control of prey? PLoS ONE 6:e20782.

Iwasa, Y., A. Pomiankowski, and S. Nee. 1991. The evolution of costly mate preferences II. The "handicap" principle. Evolution 45:1431-1442.

Johnson, M. T. J., M. Vellend, and J. R. Stinchcombe. 2009. Evolution in plant populations as a drive of ecological changes in arthropod communities. Proceedings of the Royal Society B 364:1593-1605.

Jones, L. E., and S. P. Ellner. 2004. Evolutionary tradeoff and equilibrium in an aquatic predator-prey system. Bulletin of Mathematical Biology 66:1547-1573.
Jones, L. E., and S. P. Ellner. 2007. Effects of rapid prey evolution on predator-prey cycles. Journal of Mathematical Biology 55:541-573

Jones, L. E., L. Becks, S. P. Ellner, N. G. Hairston Jr., T. Yoshida, and G. F. Fussmann. 2009. Rapid contemporary evolution and clonal food web dynamics. Philosophical Transactions of the Royal Society B 364:1579-1591.

Kondoh, M. 2003. Foraging adaptation and the relationship between food-web complexity and stability. Science 299:13881391.

Kopp, M., and W. Gabriel. 2006. The dynamic effects of an inducible defense in the Nicholson-Bailey model. Theoretical Population Biology 70:43-55.

Lande, R. 1976. Natural selection and random genetic drift in phenotypic evolution. Evolution 30:314-334.

Lande, R. 1982. A quantitative genetic theory of life history evolution. Ecology 63:607-615.

Lankau, R., and S. Y. Strauss. 2007. Mutual feedbacks maintain both genetic and species diversity in a plant community. Science 317:1561-1563.

Levin, S., and J. Udovic. 1977. A mathematical model of coevolving populations. American Naturalist 111:657-675.

Levins, R. 1974. Discussion paper: the qualitative analysis of partially specified systems. Annals of the New York Academy of Sciences 231:123-138.

Marrow, P., U. Dieckmann, and R. Law. 1996. Evolutionary dynamics of predator-prey systems: an ecological perspective. Journal of Mathematical Biology 34:556-578.

Mizoguchi, K., M. Morita, C. R. Fischer, M. Yoichi, Y. Tanji, and H. Unno. 2003. Coevolution of bacteriophage PP01 and Escherichia coli $\mathrm{O} 157: \mathrm{H} 7$ in continuous culture. Applied Environmental Microbiology 69:170-176.

Mougi, A. 2012a. Predator-prey coevolution driven by size selective predation can cause anti-synchronized and cryptic population dynamics. Theoretical Population Biology 81:113-118.

Mougi, A. 2012b. Unusual predator-prey dynamics under reciprocal phenotypic plasticity. Journal of Theoretical Biology 305:96102.

Mougi, A. 2013. Allelopathic adaptation can cause competitive coexistence. Theoretical Ecology 6:165-171.

Mougi, A., and Y. Iwasa. 2010. Evolution towards oscillation or stability in a predator-prey system. Proceedings of the Royal Society B 277:3163-3171.

Mougi, A., and Y. Iwasa. 2011. Unique coevolutionary dynamics in a predator-prey system. Journal of Theoretical Biology 277:83-89.

Nachappa, P., D. C. Margolies, J. R. Nechols, and J. F. Campbell. 2011. Variation in predator foraging behaviour changes predatorprey spatio-temporal dynamics. Functional Ecology 25:13091317.

Patel, S., and S. J. Schreiber. 2015. Evolutionary-driven shifts in communities with intraguild predation. American Naturalist 186: E98-E110.

Patel, S., M. H. Cortez, and S. J. Schreiber. 2018. Partitioning the effects of ecology, evolution, and eco-evolutionary feedbacks on community stability. American Naturalist 191:381-394.

Pruitt, J. N., and S. E. Riechert. 2009. Frequency-dependent success of cheaters during foraging bouts might limit their spread within colonies of a socially polymorphic spider. Evolution 63:29662973

Rosenzweig, M. L., and R. H. MacArthur. 1963. Graphical representation and stability conditions of predator-prey interactions. American Naturalist 97:209-223.

Saloniemi, I. 1993. A coevolutionary predator-prey model with quantitative characteristics. American Naturalist 141:880-896.

Schreiber, S. J., R. Bürger, and D. I. Bolnick. 2011. The community effects of phenotypic and genetic variation within a predator population. Ecology 92:1582-1593.

Shefferson, R. P., and R. Salguero-Gómez. 2015. Eco-evolutionary dynamics in plants: interactive processes at overlapping timescales and their implications. Journal of Ecology 103:789-797. 
Steiner, C. F., and J. Masse. 2013. The stabilizing effects of genetic diversity on predator-prey dynamics. F1000Research 2:43.

Taper, M. L., and T. J. Case. 1992. Models of character displacement and the theoretical robustness of taxon cycles. Evolution 46:317-333.

Tien, R. J., and S. P. Ellner. 2012. Variable cost of prey defense and coevolution in predator-prey systems. Ecological Monographs 82:491-504.

Turelli, M., and N. H. Barton. 1994. Genetic and statistical analyses of strong selection on polygenic traits: What, me normal? Genetics 138:913-941.

Underwood, N. 1999. The inuence of plant and herbivore characteristics on the interaction between induced resistance and herbivore population dynamics. American Naturalist 153:292-294.

Utsumi, S. 2015. Feeding evolution of a herbivore inuences an arthropod community through plants: implications for plantmediated eco-evolutionary feedback loop. Journal of Ecology 103:829-839.

Vasseur, D. A., P. Amarasekare, V. H. W. Rudolf, and J. M. Levine. 2011. Eco-evolutionary dynamics enable coexistence via neighbor-dependent selection. American Naturalist 178:E96-E109. van Velzen, E., and U. Gaedke. 2017. Disentangling eco-evolutionary dynamics of predator-prey coevolution: the case of antiphase cycles. Scientific Reports 7:17125.

Vos, M., B. W. Kooi, D. L. DeAngelis, and W. M. Mooij. $2004 a$. Inducible defences and the paradox of enrichment. Oikos 105:471-480.

Vos, M., A. M. Verschoor, B. W. Kooi, F. L. Wäckers, D. L. DeAngelis, and W. M. Mooij. 2004b. Inducible defenses and trophic structure. Ecology 85:2783-2794.

Yamamichi, M., and S. P. Ellner. 2016. Antagonistic coevolution between quantitative and Mendelian traits. Proceedings of the Royal Society B 283:20152926.

Yamamichi, M., C. L. Meunier, A. Peace, C. Prater, and M. A. Rúa. 2015. Rapid evolution of a consumer stoichiometric trait destabilizes consumer-producer dynamics. Oikos 124:690-969.

Yoshida, T., L. E. Jones, S. P. Ellner, G. F. Fussmann, and N. G. Hairston Jr. 2003. Rapid evolution drives ecological dynamics in a predator-prey system. Nature 424:303-306.

Yoshida, T., S. P. Ellner, L. E. Jones, B. J. M. Bohannan, R. E. Lenski, and N. G. Hairston Jr. 2007. Cryptic population dynamics: rapid evolution masks trophic interactions. PLoS Biology 5:e235.

\section{SUPPORTING INFORMATION}

Additional supporting information may be found online at: http://onlinelibrary.wiley.com/doi/10.1002/ecm.1304/full 